JOURNAL OF THE

AMERICAN MATHEMATICAL SOCIETY

Volume 27, Number 2, April 2014, Pages 437-477

S 0894-0347(2013)00780-8

Article electronically published on August 5, 2013

\title{
EPIPELAGIC REPRESENTATIONS AND INVARIANT THEORY
}

\author{
MARK REEDER AND JIU-KANG YU
}

\section{INTRODUCTION}

Let $k$ be a locally compact field which is complete with respect to a discrete valuation, with ring of integers $A$ and residue field $\mathfrak{f}$ of characteristic $p$. Let $K$ be a maximal unramified extension of $k$, with residue field $\mathfrak{F}$, an algebraic closure of $\mathfrak{f}$. Let $G$ be a connected reductive algebraic group over $k$. We will assume throughout this paper that $G$ splits over a tamely ramified extension of $k$. In this introduction we in fact assume $G$ is split over $K$ and that $G$ is semisimple and simply connected.

In this paper we introduce a new approach to the representation theory of $G(k)$, based on the geometric invariant theory (GIT) of Moy-Prasad filtrations of $G(K)$.

Recall that the group $G(K)$ acts on its Bruhat-Tits building $\mathcal{B}(G, K)$ and for each $x \in \mathcal{B}(G, K)$ the stabilizer $G(K)_{x}$ is a pro-algebraic group. In their foundational paper [26], Moy and Prasad constructed, for each $x \in \mathcal{B}(G, K)$, a filtration by normal subgroups of $G(K)_{x}$ :

$$
G(K)_{x}>G(K)_{x, r_{1}}>G(K)_{x, r_{2}}>\cdots,
$$

indexed by an increasing discrete sequence $\mathbf{r}(x)=\left(r_{1}, r_{2}, \ldots\right)$ of positive real numbers which depends on $x$. The quotient $\mathrm{G}_{x}:=G(K)_{x} / G(K)_{x, r_{1}}$ is a connected reductive group defined over $\mathfrak{f}$, and for $r=r_{i} \in \mathbf{r}(x)$ the quotient $\mathrm{V}_{x, r}:=$ $G(K)_{x, r} / G(K)_{x, r+}$, where $r+=r_{i+1}$, affords an algebraic representation of $\mathrm{G}_{x}$, induced by the conjugation action of $G(K)_{x}$ on $G(K)_{x, r}$.

We are interested in the GIT of $\mathrm{G}_{x}$ acting on the dual representation $\check{\mathrm{V}}_{x, r}$, for each $r \in \mathbf{r}(x)$. In this context, recall that a linear functional $\lambda \in \check{\mathbf{V}}_{x, r}$ is semistable if its orbit under $\mathrm{G}_{x}$ does not contain zero in its closure under the Zariski topology on $\check{\mathrm{V}}_{x, r}$ and $\lambda$ is stable if its orbit is closed and its stabilizer in $\mathrm{G}_{x}$ is finite.

Let $\pi$ be an irreducible admissible representation of $G(k)$ of positive depth. That is, $\pi$ has no nonzero vectors invariant under $G(k)_{y, s_{1}}$ for any $y \in \mathcal{B}(G, k)$, where $s_{1}$ is the smallest element of $\mathbf{r}(y)$. For such representations Moy and Prasad show that there are a point $x \in \mathcal{B}(G, k)$ and an $r \in \mathbf{r}(x)$ such that the space of vectors in $\pi$ fixed by $G(k)_{x, r+}$, regarded as a representation of the abelian group $\mathrm{V}_{x, r}$, contains a character of $\mathrm{V}_{x, r}$ arising from a semistable functional $\lambda \in \check{\mathrm{V}}_{x, r}$. This led them to the notion of the "depth" of $\pi$ and thence to a rough classification of the irreducible representations of $G(k)$. To sharpen this classification, it is essential to answer the following.

Received by the editors August 13, 2012 and, in revised form, June 20, 2013.

2010 Mathematics Subject Classification. Primary 22E50, 11S15, 11 S37.

The first author was supported by NSF grants DMS-0801177 and DMS-0854909.

The second author was supported by NSF grant DMS-0854909. 
Question 1. For which pairs $(x, r)$ does $\check{V}_{x, r}$ contain semistable vectors?

At the time of [26], this appeared to be a complicated problem in linear programming, and the problem has remained open until now.

The starting point of our work in this paper was the observation that stable vectors are also important for the representation theory of $G(k)$, as they can be used to give a new construction of certain supercuspidal representations of small positive depth. We will discuss this construction shortly. For the moment, suffice it to say that it became essential for us to answer the following.

Question 2. For which pairs $(x, r)$ does $\check{\mathrm{V}}_{x, r}$ contain stable vectors?

With some restrictions on $p$, we answer Questions 1 and 2 by showing that the representations $\mathrm{G}_{x} \rightarrow \mathrm{GL}\left(\check{\mathrm{V}}_{x, r}\right)$ are exactly the representations arising from gradings on semisimple Lie algebras over $\mathfrak{F}$. To explain this, we may assume, by passing to a smaller group, that $\mathbf{r}(x)=(1 / m, 2 / m, \ldots)$, for some positive integer $m$. Then the $\mathrm{G}_{x}$-modules $\mathrm{V}_{x, i / m}$ depend only on $i$ modulo $m$. On the other hand, from the Kac-Vinberg theory of periodic gradings on Lie algebras, the point $x$ also determines a $\mathbb{Z} / m$-grading $\oplus \mathfrak{L}_{i}$ on the $\mathfrak{F}$-Lie algebra $\mathfrak{L}$ whose root system is that of $G$. Assuming $p$ does not divide $m$, we show (see Theorem 4.1) that this grading coincides with the associated grading of the Moy-Prasad filtration $G(K)_{x}>G(K)_{x, 1 / m}>\cdots>G(K)_{x, 1}$. In particular $\mathfrak{L}_{0}$ is the Lie algebra of $\mathrm{G}_{x}$, which therefore acts on the summands $\mathfrak{L}_{i}$ and we have isomorphisms of representation pairs:

$$
\left(\mathrm{G}_{x}, \check{\mathrm{V}}_{x, i / m}\right) \simeq\left(\mathrm{G}_{x}, \mathfrak{L}_{i}\right)
$$

The point of (1.1) is that the GIT of the representations $\left(G_{x}, \mathfrak{L}_{i}\right)$ is very simple. This was discovered by Vinberg (over $\mathbb{C}$ ) 37] and extended to fields of good odd positive characteristic by Levy [24]. To describe their results, we may assume $i=1$. Vinberg and Levy show that the GIT of $\left(G_{x}, \mathfrak{L}_{1}\right)$ is similar to that of the adjoint representation of a reductive group. In particular, there is a Cartan subspace $\mathfrak{c} \subset \mathfrak{L}_{1}$, unique up to $\mathrm{G}_{x}$-conjugacy, and a little Weyl group $W_{\mathfrak{c}}$ such that the restriction map on invariant polynomial rings is an isomorphism

$$
\mathfrak{F}\left[\mathfrak{L}_{1}\right]^{\mathrm{G}_{x}} \stackrel{\sim}{\longrightarrow} \mathfrak{F}[\mathfrak{c}]^{W_{\mathfrak{c}}},
$$

and these rings are themselves polynomial in $\operatorname{dim} \mathfrak{c}$ homogeneneous generators. All closed $\mathrm{G}_{x}$-orbits in $\mathfrak{L}_{1}$ meet $\mathfrak{c}$, so $\mathfrak{L}_{1}$ has semistable vectors exactly when $\mathfrak{c} \neq 0$.

As preparation for this paper we have completed, in 32, the classification of stable and semistable gradings in the Vinberg-Levy theory. Via (1.1), this transfers back to the Moy-Prasad setting, resulting in the answers to Questions 1 and 2.

Both answers involve interesting aspects of the Weyl group of $G$ (and require mild restrictions on $p$ ). We refer to Theorem 8.3 for the answer to Question 1 and confine ourselves here to Question 2. Let $\mathcal{A}$ be the apartment of a maximal $K$-split torus $T \subset G$. Say that $x \in \mathcal{A}$ is a stability point if $\check{\mathrm{V}}_{x, r}$ contains stable vectors, for some $r \in \mathbf{r}(x)$. Again by passing to a smaller group we may assume that $\mathbf{r}(x)=(1 / m, 2 / m, \ldots)$, for some positive integer $m$ and that $r=1 / m$. We show (see Corollary (5.1) that $x$ is a stability point if and only if $m$ is the order of a regular elliptic element $\sigma$ in the Weyl group of $T$ and $x$ is conjugate under the affine Weyl group to the point

$$
x_{m}:=x_{0}+\frac{1}{m} \check{\rho},
$$


where $x_{0}$ is a hyperspecial point in $\mathcal{A}$ and $\check{\rho}$ is the half-sum of a set of positive co-roots of $T$. The Vinberg grading on $\mathfrak{L}$ corresponding to $x_{m}$ is given by the eigenspaces of a lift of $\sigma$ to $\operatorname{Aut}(\mathfrak{L})$.

Stability points are barycenters of certain facets in $\mathcal{A}$, and the above condition on $m$ shows that they are quite rare. Examples include the barycenter of an alcove (an open facet in $\mathcal{A}$ ). In type $A_{n}$ these are the only stability points, but in every other type there are stability points which are barycenters of facets in the boundary of an alcove. For example, in $E_{8}$ there are twelve conjugacy classes of stability points $x \in \mathcal{A}$ (see Section 5.1) corresponding to the twelve elliptic regular classes in $W\left(E_{8}\right)$.

We turn now to supercuspidal representations. Assume that $x \in \mathcal{A} \cap \mathcal{B}(G, k)$ and that we are given a stable functional $\lambda \in \check{\mathrm{V}}_{x, r_{1}}(\mathfrak{f})$, with isotropy group $\mathrm{A}_{\lambda} \subset \mathrm{G}_{x}$. With this data we construct (see Section 2.5) a family of irreducible supercuspidal representations $\pi_{x}(\lambda, \rho)$ of $G(k)$, parameterized by irreducible representations $\rho$ of a certain finite-dimensional semisimple algebra $\mathcal{H}_{\lambda}$ (which in most cases is the group algebra of $A_{\lambda}(\mathfrak{f})$ ). These are the "epipelagic representations" in the title 1 They have depth $r_{1}$, the smallest element of $\mathbf{r}(x)$.

If $x$ is the barycenter of an alcove, then the $\pi_{x}(\lambda, \rho)$ are the "simple supercuspidal representations" of [14], of depth $1 / h$, where $h$ is the Coxeter number of $G$. (For $G$ of type $A_{n}$, such representations were constructed by Carayol [10.) In this case the group $\mathrm{G}_{x}$ is a torus and stable functionals are just those with nonzero component in each weight space. When $x$ is contained in the boundary of an alcove, as in the $E_{8}$ examples mentioned above, the group $\mathrm{G}_{x}$ is no longer a torus, and the GIT of its action on $\check{\mathrm{V}}_{x, r_{1}}$ becomes the essential point in the construction. For such points $x$, the epipelagic representations in this paper have no analogue in type $A_{n}$.

If $p>h$, then one can also construct our epipelagic representations following Adler [1. (The generalization of Adler's approach in 38. gives all supercuspidal representations of $G(k)$ when $p$ is large [20].) To implement Adler's method for epipelagic representations, one must construct a certain tamely ramified anisotropic torus in $G(k)$ having $x$ as its unique fixed point in $\mathcal{B}(G, k)$. This was carried out by Kaletha [18 in work based on an earlier version of this paper. In contrast, the GIT approach used here does not require any restrictions on $p$; it works even when the above tamely ramified torus does not exist. Instead, the required input is a stable rational vector in $\check{\mathrm{V}}_{x, r_{1}}$.

Epipelagic representations have already found other applications to automorphic forms [13], 16], 21], the explicit Langlands correspondence [9], and inverse Galois theory [19]. Given a stable point in $\check{\mathrm{V}}_{x, r_{1}}$, the construction of the corresponding epipelagic representations is very simple and does not require the full structure theory of $G$. For this reason we describe our construction early in the paper, with a minimum of technicalities, in order to make the epipelagic construction more accessible to nonspecialists.

Finally, it is natural to ask about the Langlands parameters corresponding to our epipelagic representations $\pi_{x}(\lambda, \rho)$. If $p>h$, a stable functional $\lambda \in \check{\mathrm{V}}_{x, 1}$ leads naturally to a discrete Langlands parameter

$$
\varphi_{\lambda}: \mathcal{W}_{k} \longrightarrow{ }^{L} G
$$

\footnotetext{
${ }^{1}$ In oceanography, the epipelagic zone is the uppermost layer of the open sea where photosynthesis can occur.
} 
from the Weil group of $k$ to the $L$-group of $G$, whose image of Frobenius is determined by the $\mathfrak{f}$-structure on a Cartan subspace of $\check{\mathrm{V}}_{x, r_{1}}$ containing $\lambda$. The inertia subgroup $\mathcal{I}_{k} \leq \mathcal{W}_{k}$ maps to the normalizer of a maximal torus in ${ }^{L} G$, and the projection of $\varphi_{\lambda}\left(\mathcal{I}_{k}\right)$ to the Weyl group of ${ }^{L} G$ is generated by the elliptic $\mathbb{Z}$-regular element $\sigma$ corresponding to $x$, as above.

This parameter $\varphi_{\lambda}$ satisfies properties expected of the local Langlands conjecture (see [14, for example). First, $\varphi_{\lambda}$ is itself "epipelagic", in the sense that the upper ramification filtration $\varphi_{\lambda}\left(\mathcal{W}_{k}\right)$ has a single upper break at $r_{1}$, equal to the depth of the representations $\pi_{x}(\lambda, \rho)$. Second, the adjoint Swan conductor of $\varphi_{\lambda}$ is equal to the dimension of $\mathrm{G}_{x}$, and the centralizer of $\varphi_{\lambda}$ in the dual group $\hat{G}$ has the same cardinality as the isotropy group $\mathrm{A}_{\lambda}(\mathfrak{f})$. It follows that the Euler-Poincaré formal degree [14, 7.1] of $\pi_{x}(\lambda, \rho)$ is given in terms of the adjoint gamma value $\gamma\left(\varphi_{\lambda}, \hat{\mathfrak{g}}, 0\right)$ as proposed in [17] (see also [14]).

If $p$ is small, then (1.1) does not immediately apply. In many cases, however, one can still find stable points in $\check{V}_{x, r_{1}}(\mathfrak{f})$, hence epipelagic representations. In Sections 7.4 and 7.5 we illustrate the epipelagic construction at these interesting primes $p$, and we again construct candidates for their Langlands parameters. Here, though the epipelagic representations have a uniform construction, the parameters depend crucially on the particular prime $p$, and the depth of the representation no longer agrees with the depth of the parameter. This different behavior at small primes was observed already in [14.

\section{Supercuspidal RePRESEnTAtions}

2.1. Compact induction. We will review here some results on compact induction which are well known to the experts. See [8] for more details.

Let $G$ be a locally profinite group with center $Z$. Let $H$ be an open subgroup of $G$ such that $Z \subset H$ and $H / Z$ is compact, and let $\tau$ be a smooth finite-dimensional representation of $H$ on a complex vector space $W$. The compactly induced representation $\operatorname{ind}_{H}^{G} \tau$ is realized on the complex vector space of functions $f: G \rightarrow W$ satisfying the following two conditions:

- $f(h x)=\tau(h) f(x)$ for all $h \in H$ and $x \in G$;

- $f$ is supported on only finitely many cosets of $H$ in $G$.

The group $G$ acts on $\operatorname{ind}_{H}^{G} \tau$ by right translations: $[g \cdot f](x)=f(x g)$.

For $g \in G$, let $H^{g}=g^{-1} H g$ and let $\tau^{g}: H^{g} \rightarrow G L(W)$ be the conjugate representation, given by $\tau^{g}\left(h^{g}\right)=\tau(h)$. The intertwining of $\tau$ is the set $I(G, H, \tau)=$ $\left\{g \in G: \tau \simeq \tau^{g}\right.$ on $\left.H \cap H^{g}\right\}$. Note that $I(G, H, \tau)$ is preserved under leftmultiplication by $H$ and hence is a union of cosets of $H$ in $G$.

Now assume that $G$ is the group of $k$-rational points in a connected reductive algebraic group defined over a field $k$ which is complete and locally compact, with respect to a discrete valuation. A proof of the following basic result can be found in [8, 3.11.4].

Lemma 2.1. Let $H$ be an open subgroup of $G$ containing the center $Z$ of $G$ with $H / Z$ compact, and let $\tau$ be an irreducible smooth representation of $H$. Then the representation $\operatorname{ind}_{H}^{G} \tau$ is irreducible for $G$ if and only if $I(G, H, \tau)=H$.

If $J<H$ are two open subgroups of $G$ containing $Z$ and compact modulo $Z$ and $\tau$ is a smooth representation of $J$, then $\operatorname{ind}_{J}^{H} \tau$ is smooth and we have transitivity 
of induction:

$$
\operatorname{ind}_{J}^{G} \tau \simeq \operatorname{ind}_{H}^{G} \operatorname{ind}_{J}^{H} \tau \text {. }
$$

Suppose in addition that $J$ is normal in $H$, and let $\chi: J \rightarrow \mathbb{C}^{\times}$be a smooth character of $J$. If $h \in H$, then $\chi^{h}$ is again a character of $J$, possibly equal to $\chi$, and $I(G, J, \chi)$ is a union of cosets of the stabilizer $H_{\chi}=\left\{h \in H: \chi^{h}=\chi\right\}$. We have $J \unlhd H_{\chi}$ and the quotient $A_{\chi}:=H_{\chi} / J$ is a finite group.

From Mackey theory for finite groups, we deduce the following. The intertwining algebra

$$
\mathcal{H}_{\chi}:=\operatorname{End}_{H_{\chi}}\left(\operatorname{ind}_{J}^{H_{\chi}} \chi\right)
$$

has dimension equal to $\left|A_{\chi}\right|$, and we have a bijection, denoted $\rho \mapsto \chi_{\rho}$, from the set $\operatorname{Irr}\left(\mathcal{H}_{\chi}\right)$ of simple $\mathcal{H}_{\chi}$-modules (up to equivalence) to the set of irreducible constituents of $\operatorname{ind}_{J}^{H_{\chi}} \chi$ such that

$$
\operatorname{ind}_{J}^{H_{\chi}} \chi=\bigoplus_{\rho \in \operatorname{Irr}\left(\mathcal{H}_{\chi}\right)}(\operatorname{dim} \rho) \cdot \chi_{\rho} .
$$

Finally, each $\chi_{\rho}$ is $\chi$-isotypic when restricted to $J$ and induces irreducibly to $H$.

Lemma 2.2. Assume that $I(G, J, \chi)=H_{\chi}$. Then the following hold.

(1) The representation $\operatorname{ind}_{J}^{G} \chi$ has a finite direct sum decomposition

$$
\operatorname{ind}_{J}^{G} \chi=\bigoplus_{\rho \in \operatorname{Irr}\left(\mathcal{H}_{\chi}\right)}(\operatorname{dim} \rho) \cdot \operatorname{ind}_{H_{\chi}}^{G} \chi_{\rho} .
$$

(2) For each $\rho \in \operatorname{Irr}\left(\mathcal{H}_{\chi}\right)$, the compactly induced representation $\operatorname{ind}_{H_{\chi}}^{G} \chi_{\rho}$ is irreducible.

(3) If $\rho, \rho^{\prime}$ are inequivalent simple modules for $\mathcal{H}_{\chi}$, then $\operatorname{ind}_{H_{\chi}}^{G} \chi_{\rho}$ and $\operatorname{ind}_{H_{\chi}}^{G} \chi_{\rho^{\prime}}$ are inequivalent representations of $G$.

Proof. The direct sum decomposition (2.2) follows from (2.1) and transitivity of induction.

To see that the summands are irreducible, we consider their intertwining. Suppose $g \in I\left(G, H_{\chi}, \chi_{\rho}\right)$. This means $\chi_{\rho}=\left(\chi_{\rho}\right)^{g}$ on $H_{\chi} \cap H_{\chi}^{g}$. Restricting to $J$, we get $\chi=\chi^{g}$ on $J \cap J^{g}$, so that $g \in I(G, J, \chi)$, which is equal to $H_{\chi}$ by assumption. Hence $I\left(G, H_{\chi}, \chi_{\rho}\right)=H_{\chi}$, so $\operatorname{ind}_{H_{\chi}}^{G} \chi_{\rho}$ is irreducible, by Lemma 2.1.

A variant of Lemma 2.1 can be used to show inequivalence, or one can argue as follows. From Mackey theory for $G$ [23] and Frobenius reciprocity [8, 1.2.5], it follows that

$$
\operatorname{End}_{G}\left(\operatorname{ind}_{J}^{G} \chi\right) \simeq \bigoplus_{g \in J \backslash G / J} \operatorname{Hom}_{J \cap J^{g}}\left(\chi, \chi^{g}\right)=\bigoplus_{g \in A_{\chi}} \operatorname{Hom}_{J}(\chi, \chi),
$$

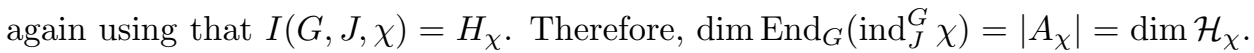
It follows that there can be no nonzero intertwining maps between summands for $\rho \neq \rho^{\prime}$ in (2.2). This completes the proof.

Remark 1. If $\chi$ extends to a character $\tilde{\chi}$ on $H_{\chi}$, then $\mathcal{H}_{\chi}$ is just the group algebra of $A_{\chi}$. Each $\rho \in \operatorname{Irr}\left(A_{\chi}\right)$ may be viewed as a representation of $H_{\chi}$ via the projection $H_{\chi} \rightarrow A_{\chi}$, and we may take $\chi_{\rho}=\tilde{\chi} \otimes \rho$.

In our applications of Lemma 2.2, the group $J$ will always be a pro- $p$ group. In most cases, $A_{\chi}$ will have order prime to $p$, so the quotient $H_{\chi} \rightarrow A_{\chi}$ will split and 
$\chi$ will extend to $H_{\chi}$. We can also extend $\chi$ whenever $H^{2}\left(A_{\chi}, \mathbb{C}^{\times}\right)=1$, for example if $A_{\chi}$ is cyclic or symmetric on three letters.

Remark 2. The formal degree of $\operatorname{ind}_{H_{\chi}}^{G} \chi_{\rho}$ with respect to a Haar measure $\mu$ on $G$ is given by

$$
\operatorname{deg}_{\mu}\left(\operatorname{ind}_{H_{\chi}}^{G} \chi_{\rho}\right)=\frac{\operatorname{dim} \chi_{\rho}}{\mu\left(H_{\chi}\right)}=\frac{\operatorname{dim} \chi_{\rho}}{\left|A_{\chi}\right|} \cdot \frac{1}{\mu(J)} .
$$

2.2. Nonarchimedean local fields. Let $k$ be a locally compact field which is complete with respect to a discrete valuation $\mathrm{v}$, with value group $\mathrm{v}\left(k^{\times}\right)=\mathbb{Z}$. Let $A=\{a \in k: \vee(a) \geq 0\}$ denote the ring of integers of $k$, and let $p$ be the characteristic of the residue field $\mathfrak{f}$ of $k$.

We fix a separable closure $\bar{k}$ of $k$ and let $K$ be the maximal unramified extension of $k$ in $\bar{k}$. The valuation $v$ extends to $K$, again taking values in $\mathbb{Z}$. The residue field $\mathfrak{F}$ of $K$ is an algebraic closure of $\mathfrak{f}$.

2.3. Semisimple groups. For the rest of the paper, $G$ denotes a connected semisimple algebraic group defined over $k$. Let $S$ be a maximal $k$-split torus in $G$ and let $T$ be a maximal $K$-split torus of $G$ containing $S$. We may and shall choose $T$ to be defined over $k$ [7, 5.1.12]. Let $F: G(K) \rightarrow G(K)$ be the Frobenius action arising from the given $k$-structure on $G$; the constructions in Section 2.1 will be applied to the locally profinite group of $k$-rational points: $G(k)=G(K)^{F}$.

Let $\mathcal{A}(T, K)$ be the apartment of $T$ in the Bruhat-Tits building $\mathcal{B}(G, K)$ of $G(K)$ and let $\Psi_{K}$ denote the set of affine $K$-roots of $T$ in $G$. Each $\psi \in \Psi_{K}$ is an affine function on $\mathcal{A}(T, K)$ whose gradient $\dot{\psi}$ is a $K$-root of $T$ in $G$. We have $\dot{\psi}_{1}=\dot{\psi}_{2}$ if and only if $\psi_{1}-\psi_{2}$ is a constant function.

The Frobenius map $F$ extends to a mapping on $\mathcal{B}(G, K)$ which preserves and acts affinely on $\mathcal{A}(T, K)$. We may identify

$$
\mathcal{B}(G, k)=\mathcal{B}(G, K)^{F} \quad \text { and } \quad \mathcal{A}(S, k)=\mathcal{A}(T, K)^{F},
$$

where $\mathcal{B}(G, k)$ is the building of $G(k)$ and $\mathcal{A}(S, k)$ is the apartment in $\mathcal{B}(G, k)$ corresponding to $S$ [36, 2.6.1].

Each affine $K$-root in $\Psi_{K}$ restricts to an affine function (possibly the zero function) on $\mathcal{A}(S, k)$. We let $\mathcal{A}(T, K)_{\mathbb{Q}}$ and $\mathcal{A}(S, k)_{\mathbb{Q}}$ be the subsets of the respective apartments where all roots in $\Psi_{K}$ take rational values.

For $x \in \mathcal{A}(T, K)$ and $r \geq 0$, let

$$
T(K)_{r}, \quad T(K)_{r+}, \quad G(K)_{x, r}, \quad G(K)_{x, r+}
$$

denote the filtration groups defined by Moy and Prasad [26]. For example, $G(K)_{x, 0}$ is the parahoric subgroup of $G(K)$ attached to $x$ by Bruhat-Tits theory and $G(K)_{x, 0+}$ is the pro-unipotent radical of $G(K)_{x, 0}$. The top quotient

$$
\mathrm{G}_{x}:=G(K)_{x, 0} / G(K)_{x, 0+}
$$

is a connected reductive group over the residue field $\mathfrak{F}$. For $r>0$ the quotient

$$
\mathrm{\vee}_{x, r}:=G(K)_{x, r} / G(K)_{x, r+}
$$

is abelian and is in fact a finite-dimensional vector space over $\mathfrak{F}$. The conjugation action of $G(K)_{x, 0}$ on $G(K)_{x, r}$ induces an algebraic representation of $\mathrm{G}_{x}$ on $\mathrm{V}_{x, r}$. If $x \in \mathcal{A}(S, k)$, then both $\mathrm{G}_{x}$ and its representation $\mathrm{V}_{x, r}$ are defined over $\mathfrak{f}$, with Frobenius action induced by $F$. 
The quotient

$$
\mathrm{T}:=T(K)_{0} / T(K)_{0+}
$$

is a maximal torus in $\mathrm{G}_{x}$, whose character group may be identified with that of $T$. The nonzero weights of $\mathrm{T}$ in $\mathrm{V}_{x, r}$ are the gradients $\dot{\psi}$ where $\psi(x)=r$.

2.4. Stable vectors. Let $\check{\mathrm{V}}_{x, r}=\operatorname{Hom}\left(\mathrm{V}_{x, r}, \mathfrak{F}\right)$ be the representation of $\mathrm{G}_{x}$ dual to $\mathrm{V}_{x, r}$. Following [28, we say that a functional $\lambda \in \check{\mathrm{V}}_{x, r}$ is stable if the following two conditions hold:

- the orbit $\mathrm{G}_{x} \cdot \lambda$ is Zariski-closed in $\check{\mathrm{V}}_{x, r}$ and

- the stabilizer of $\lambda$ in $\mathrm{G}_{x}$ is finite, as an algebraic group.

Given two points $x, y \in \mathcal{A}(T, K)_{\mathbb{Q}}$ and a rational number $r>0$, the image $\mathrm{V}_{x, y, r}$ of $G(K)_{y, r+} \cap G(K)_{x, r}$ in $\mathrm{V}_{x, r}$ is a $\mathrm{T}$-stable subspace of $V_{x, r}$ with weight decomposition

$$
\mathrm{V}_{x, y, r}:=\bigoplus_{\substack{\psi \in \Psi_{x, r} \\ \psi(y)>r}} \mathrm{~V}_{x, r}(\dot{\psi}) .
$$

The key step for constructing epipelagic representations is the following.

Lemma 2.3. Let $\lambda \in \check{\mathrm{V}}_{x, r}$ be a stable functional. If $\lambda$ vanishes identically on $\mathrm{V}_{x, y, r}$, then $y=x$.

Proof. Let $X_{*}(T)$ be the co-character group of $T$. The vector space $\mathbb{Q} \otimes X_{*}(T)$ acts transitively on $\mathcal{A}(T, K)_{\mathbb{Q}}$ by translations, so the difference $v=y-x$ lies in $\mathbb{Q} \otimes X_{*}(T)$. Assume that $v \neq 0$. Then we can write $v=s \gamma$, where $\gamma \in X_{*}(T)$ and $s$ is a positive rational number. For all $\psi \in \Psi_{x, r}$, we have

$$
\psi(y)=\psi(x+s \gamma)=\psi(x)+s\langle\dot{\psi}, \gamma\rangle=r+s\langle\dot{\psi}, \gamma\rangle .
$$

Since $s>0$, the weight decomposition of $\mathrm{V}_{x, y, r}$ is

$$
\mathrm{V}_{x, y, r}=\bigoplus_{\substack{\psi \in \Psi_{x, r} \\\langle\dot{\psi}, \gamma\rangle>0}} \mathrm{~V}_{x, r}(\dot{\psi}) .
$$

The weight decomposition of $\lambda$ is of the form

$$
\lambda=\sum_{\psi \in \Psi_{x, r}} \lambda_{-\dot{\psi}}
$$

where $\lambda_{-\dot{\psi}}$ has $\mathrm{T}$-weight $-\dot{\psi}$. If $\lambda$ vanishes identically on $\mathrm{V}_{x, y, r}$, then $\lambda_{-\dot{\psi}}=0$ if $\langle\dot{\psi}, \gamma\rangle>0$, so we have

$$
\lambda=\mu+\sum_{\substack{\psi \in \Psi_{x, r} \\\langle\dot{\psi}, \gamma\rangle<0}} \lambda_{-\dot{\psi}},
$$

where

$$
\mu=\sum_{\substack{\psi \in \Psi_{x, r} \\\langle\dot{\psi}, \gamma\rangle=0}} \lambda_{-\dot{\psi}}
$$

Since

$$
\gamma(t) \cdot \lambda=\mu+\sum_{\substack{\psi \in \Psi_{x, r} \\\langle\dot{\psi}, \gamma\rangle<0}} t^{-\langle\dot{\psi}, \gamma\rangle} \lambda_{-\dot{\psi}}
$$


it follows that

$$
\lim _{t \rightarrow 0} \gamma(t) \cdot \lambda=\mu \text {. }
$$

Since the $\mathrm{G}_{x}$-orbit of $\lambda$ is closed, we have $\mu \in \mathrm{G}_{x} \cdot \lambda$, so $\mu$ is also a stable vector in $\check{\mathrm{V}}_{x, r}$. But $\gamma$ takes values in the stabilizer of $\mu$ in $\mathrm{G}_{x}$, which is finite, so $\gamma=0$, contradicting the assumption that $y \neq x$.

Remark. If we assume $x, y \in \mathcal{A}(S, k)_{\mathbb{Q}}$, then the proof of Lemma 2.3 requires only the weaker assumption that the stabilizer of $\lambda$ in $G_{x}$ is anisotropic over $\mathfrak{f}$ (cf. [27, Cor. 4.9]). We will not use this variant of the result.

2.5. Epipelagic supercuspidal representations. For a given point $x \in \mathcal{A}(S, k)$, let $r(x)$ be the smallest positive value in the set $\left\{\psi(x): \psi \in \Psi_{K}\right\}$ of values of all affine $K$-roots. Thus, we have $G(K)_{x, 0+}=G(K)_{x, r(x)}$. We say that an irreducible representation $\pi$ of $G(k)$ is epipelagic if it has a nonzero vector fixed under the subgroup $G(k)_{x, r(x)+}$ and $\pi$ has depth $r(x)$. In this section we show how stable points in $\check{V}_{x, r(x)}(\mathfrak{f})$ give rise to epipelagic supercuspidal representations of $G(k)$.

We will abbreviate

$$
\begin{gathered}
J_{x}:=G(k)_{x, r(x)}, \quad J_{x}^{+}:=G(k)_{x, r(x)+} \\
\mathrm{V}_{x}:=\mathrm{V}_{x, r(x)}, \quad \check{\mathrm{V}}_{x}:=\check{\mathrm{V}}_{x, r(x)}, \quad \mathrm{V}_{x, y}=\mathrm{V}_{x, y, r(x)} .
\end{gathered}
$$

The isotropy group $G(K)_{x}:=\operatorname{Stab}_{G(K)}(x)$ contains $G(K)_{x, 0}$ with finite index. The actions of $G(K)_{x, 0}$ on $\mathrm{V}_{x}$ and $\check{\mathrm{V}}_{x}$ extend to $G(K)_{x}$ which preserves the set of $\mathrm{G}_{x}$-stable functionals in $\check{\mathrm{V}}_{x}$.

Let $\lambda \in \check{\mathbf{V}}_{x}(\mathfrak{f})$ be an $\mathfrak{f}$-rational stable functional with stabilizer $\operatorname{Stab}_{G(K)_{x}}(\lambda)$ in $G(K)_{x}$, and set

$$
H_{x}:=G(K)_{x}^{F}=G(k)_{x}, \quad H_{x, \lambda}:=\operatorname{Stab}_{G(K)_{x}}(\lambda)^{F}=\operatorname{Stab}_{H_{x}}(\lambda) .
$$

Since $\lambda$ has finite stabilizer in $\mathrm{G}_{x}$, it follows that the quotient group $\operatorname{Stab}_{G(K)_{x}}(\lambda) /$ $G(K)_{x, r(x)}$ is finite. We set

$$
A_{x, \lambda}:=H_{x, \lambda} / J_{x}=\left[\operatorname{Stab}_{G(K)_{x}}(\lambda) / G(K)_{x, r(x)}\right]^{F},
$$

where the latter equality follows from the fact that $G(K)_{x, r(x)}$ has trivial $\operatorname{Gal}(K / k)$ cohomology, by the pro-finite version of the Lang-Steinberg theorem.

Fix a nontrivial character $\chi: \mathfrak{f}^{+} \rightarrow \mathbb{C}^{\times}$of the additive group of $\mathfrak{f}$. The composition

$$
\chi_{\lambda}:=\chi \circ \lambda: \bigvee_{x}(\mathfrak{f}) \rightarrow \mathbb{C}^{\times}
$$

is a character of $J_{x}$ which is trivial on $J_{x}^{+}$, and $H_{x, \lambda}$ is the stabilizer of $\chi_{\lambda}$ in $H_{x}$. We consider the compactly induced representation

$$
\pi_{x}(\lambda):=\operatorname{ind}_{J_{x}}^{G(k)} \chi_{\lambda}
$$

and the intertwining algebra

$$
\mathcal{H}_{x, \lambda}:=\operatorname{End}_{H_{x, \lambda}}\left(\operatorname{ind}_{J_{x}}^{H_{x, \lambda}} \chi_{\lambda}\right) .
$$

If $\rho$ is a simple $\mathcal{H}_{x, \lambda}$-module, let $\chi_{\lambda, \rho}$ denote the corresponding irreducible constitutent for $H_{x, \lambda}$ in $\operatorname{ind}_{J_{x}}^{H_{x, \lambda}} \chi_{\lambda}$, as in (2.1). 
Proposition 2.4. Suppose that $\lambda \in \check{\mathfrak{V}}_{x}(\mathfrak{f})$ is an $\mathfrak{f}$-rational stable functional for the action of $\mathrm{G}_{x}$ on $\check{\mathrm{V}}_{x}$. Then the following hold.

(1) The representation $\pi_{x}(\lambda)$ has a finite direct sum decomposition

$$
\pi_{x}(\lambda)=\bigoplus_{\rho \in \operatorname{Irr}\left(\mathcal{H}_{x, \lambda}\right)} \operatorname{dim} \rho \cdot \pi_{x}(\lambda, \rho),
$$

where $\pi_{x}(\lambda, \rho):=\operatorname{ind}_{H_{x, \lambda}}^{G(k)} \chi_{\lambda, \rho}$ is an irreducible supercuspidal representation of $G(k)$, for each $\rho \in \operatorname{Irr}\left(\mathcal{H}_{x, \lambda}\right)$.

(2) If $\rho$ and $\rho^{\prime}$ are inequivalent simple modules for $\mathcal{H}_{x, \lambda}$, then $\pi_{x}(\lambda, \rho)$ and $\pi_{x}\left(\lambda, \rho^{\prime}\right)$ are inequivalent representations of $G(k)$.

(3) The formal degree of $\pi_{x}(\lambda, \rho)$ with respect to a Haar measure $\mu$ on $G(k)$ is given by

$$
\operatorname{deg}_{\mu}\left(\pi_{x}(\lambda, \rho)\right)=\frac{\operatorname{dim} \chi_{\lambda, \rho}}{\left|A_{x, \lambda}\right|} \cdot \frac{1}{\mu\left(J_{x}\right)} .
$$

Proof. By Lemma 2.2 (taking $J=J_{x}$ and $H=H_{x}$, so that $H_{\chi_{\lambda}}=H_{x, \lambda}$ ), it suffices to show that if $g \in G(k)$ and

$$
\chi_{\lambda}=\chi_{\lambda}^{g} \quad \text { on } \quad J_{x} \cap J_{x}^{g},
$$

then $g \in H_{x}$. Since $H_{x} \supset G(k)_{x, 0}$ and the latter contains an Iwahori subgroup of $G(k)$, we may write $g=a n b$, where $n \in G(k)$ normalizes $S$ and $a, b \in H_{x}$ [36, 3.3.1]. Since $J_{x}$ is normal in $H_{x}$, condition (2.6) is equivalent to

$$
{ }^{b} \chi_{\lambda}=\left(\chi_{\lambda}^{a}\right)^{n} \quad \text { on } \quad J_{x} \cap J_{x}^{n},
$$

so we certainly have

$$
{ }^{b} \chi_{\lambda}=\left(\chi_{\lambda}^{a}\right)^{n} \quad \text { on } \quad J_{x} \cap\left(J_{x}^{+}\right)^{n} .
$$

But $J_{x}^{+}$is also normal in $H_{x}$ and $\chi_{\lambda}$ is trivial on $J_{x}^{+}$, so $\left(\chi_{\lambda}^{a}\right)^{n}$ is trivial on $\left(J_{x}^{+}\right)^{n}$. Thus, (2.8) implies that ${ }^{b} \chi_{\lambda}$ is trivial on $J_{x} \cap\left(J_{x}^{+}\right)^{n}$.

Now $\mathrm{V}_{x}(\mathfrak{f})=J_{x} / J_{x}^{+}$and the image of $J_{x} \cap\left(J_{x}^{+}\right)^{n}$ in $\mathrm{V}_{x}(\mathfrak{f})$ is the subspace $\mathrm{V}_{x, y}(\mathfrak{f})$, where $y=n^{-1} \cdot x$. Moreover ${ }^{b} \chi_{\lambda}=\chi_{b \cdot \lambda}$, and $b \cdot \lambda$ is a stable vector in $\check{\bigvee}_{x}(\mathfrak{f})$ which vanishes on $\mathrm{V}_{x, y}(\mathfrak{f})$. Since $\mathrm{V}_{x, y}(\mathfrak{f})$ contains an $\mathfrak{F}$-basis of $\mathrm{V}_{x, y}$, it follows that $b \cdot \lambda$ vanishes on $\mathrm{V}_{x, y}$. Now Lemma 2.3 implies that $y=x$, so that $n \in H_{x}$. It follows that $g=a n b \in H_{x}$, as we wished to show.

2.6. Example: Simple supercuspidal representations. The above construction of supercuspidal representations requires the existence of stable orbits. For certain points $x \in \mathcal{A}(S, k)$, such orbits do indeed exist and can be found in a uniform way for all tamely ramified groups. Here we assume for convenience that $G$ is absolutely simple. Let $E_{0}$ be the smallest tamely ramified extension of $K$ splitting $G$. The Galois group $\Gamma_{0}=\operatorname{Gal}\left(E_{0} / K\right)$ is cyclic of order $e:=\left[E_{0}: K\right]$. Since $G$ is quasi-split over $K$, a generator of $\operatorname{Gal}\left(E_{0} / K\right)$ acts on the based root datum $(X, \Delta, \check{R}, \check{\Delta})$ of $G$ via an automorphism $\vartheta \in \operatorname{Aut}(R, \Delta)$ of order $e$. (See Section 3.1 for more details on the root datum and Galois action.)

An alcove in $\mathcal{A}(T, K)$ is a connected component of the set of points in $\mathcal{A}(T, K)$ on which no affine $K$-root vanishes. There exists and we choose an alcove $\mathcal{C}$ in $\mathcal{A}(T, K)$ for which $F(\mathcal{C})=\mathcal{C}$ [36, 3.4.3]. Each hyperplane bounding $\mathcal{C}$ is the zero locus of a unique affine $K$-root which is positive on $\mathcal{C}$. Let $\Pi_{\mathcal{C}}=\left\{\psi_{0}, \psi_{1}, \ldots, \psi_{\ell_{\vartheta}}\right\}$ 
be the set of these affine $K$-roots, where $\ell_{\vartheta}=\operatorname{dim} \mathcal{A}(T, K)$ is the $K$-rank of $G$. The affine roots in $\Pi_{\mathcal{C}}$ satisfy a single linear relation

$$
\sum_{i=0}^{\ell_{\vartheta}} b_{i} \psi_{i}=\frac{1}{e},
$$

where the numbering is chosen so that $b_{0}=1$ (see also Section 3.3). All of the $b_{i}$ are positive integers and their sum is

$$
\sum_{i=0}^{\ell_{\vartheta}} b_{i}=\frac{h_{\vartheta}}{e},
$$

where $h_{\vartheta}$ is the twisted Coxeter number of $(R, \vartheta)$ (cf. 30]). If $\vartheta=1$ (i.e., if $G$ is $K$-split), then $h_{\vartheta}$ is the usual Coxeter number of $G$.

The barycenter of $\mathcal{C}$ is the unique point $x \in \mathcal{C}$ at which all $\psi_{i} \in \Pi_{\mathcal{C}}$ take the same value. This common value must be $1 / h_{\vartheta}$, by equation (2.10). From the uniqueness of $x$ we have $F(x)=x$, so in fact $x \in \mathcal{A}(S, k)$.

It follows that the minimal positive value $r(x)$ (see Section [2.5) is given by

$$
r(x)=\frac{1}{h_{\vartheta}} .
$$

The subgroup $H_{x}$ is the normalizer of the Iwahori subgroup of $G(k)$ attached to $\mathcal{C}$ and $J_{x}$ is the pro-unipotent radical of $H_{x}$. The group $\mathrm{G}_{x}$ is the torus $\mathrm{T}$ whose character group $X^{*}(\mathrm{~T})=X_{\vartheta}$ is the coinvariant group $X /(1-\vartheta) X$ modulo torsion, and $\check{V}_{x}$ decomposes under $T$ into a direct sum of lines

$$
\check{\mathrm{V}}_{x}=\bigoplus_{i=0}^{\ell_{\vartheta}} \check{\mathrm{V}}_{x}\left(-\dot{\psi}_{i}\right),
$$

where $\check{\mathrm{V}}_{x}\left(-\dot{\psi}_{i}\right)$ affords the character $-\dot{\psi}_{i}$. A functional $\lambda \in \check{\mathrm{V}}_{x}$ is stable for $\mathrm{T}$ precisely when each of its components $\lambda_{i} \in \check{\mathrm{V}}_{x}\left(-\dot{\psi}_{i}\right)$ is nonzero. In this case the stabilizer of $\lambda$ in $T$ is the intersection of root kernels

$$
\mathrm{T}_{\lambda}=\bigcap_{i=0}^{\ell_{\vartheta}} \operatorname{ker}\left(\dot{\psi}_{i}\right) .
$$

Thus, $G$ has epipelagic representations of depth $1 / h_{\vartheta}$, associated to the barycenter $x$ of an alcove in $\mathcal{A}(T, K)$.

If $G$ is simply connected, then $H_{x, \lambda}=Z(k) \cdot J_{x}$, where $Z(k)$ is the center of $G(k)$, so there are $|Z(k)|$ of these representations, one with each central character. If $G$ is also split over $k$, these are the simple supercuspidal representations constructed in 14 .

\section{Semistable Points AND BARYCEnters}

We have just seen that if $x$ is the barycenter of an alcove in $\mathcal{A}(T, K)$, then $\mathrm{G}_{x}$ has stable orbits in $\check{\mathrm{V}}_{x, 1 / h_{\vartheta}}$. In this section we give a necessary condition on a rational point $x \in \mathcal{A}(T, K)$ for $\mathrm{G}_{x}$ to have semistable orbits in $\check{\mathrm{V}}_{x, r(x)}$; we will show that this can hold only if $x$ is the barycenter of some facet in $\mathcal{A}(T, K)$.

There are still no restrictions on the residue characteristic $p$ other than $G$ being split over a tamely ramified extension of $k$. For convenience we assume in this section that $G$ is absolutely simple. 
3.1. Root data. We now require a more detailed account of the structure of $G$. Recall that $\bar{k}$ is a fixed separable closure of $k$ and that $E_{0} \subset \bar{k}$ is the minimal tame extension of $K$ which splits $G$. Let $e$ be the degree of $E_{0} / K$ and choose a generator $\sigma_{0}$ of the Galois group $\operatorname{Gal}\left(E_{0} / K\right)$.

Recall that $S$ is a maximal $k$-split torus in $G$, contained in a maximal $K$-split $k$ torus of $G$ defined over $k$. Since $G$ is quasi-split over $K$, the centralizer $M=C_{G}(T)$ is a maximal torus of $G$ contained in a $K$-rational Borel subgroup $B$ of $G$. Let $N=N_{G}(M)$ be the normalizer of $M$ in $G$, and let $W=N / M$ be the absolute Weyl group of $G$.

The based root datum of the split group $G \times{ }_{k} E_{0}$ is a quadruple $\Phi:=(X, \Delta, \check{X}, \check{\Delta})$, where $X=X^{*}(M)$ and $\check{X}=X_{*}(M)$ are the character and co-character groups of $M$ and where $\Delta$ and $\check{\Delta}$ are the simple roots and co-roots of $M$ in $B$. The latter are contained in the sets $R$ and $\check{R}$ of all roots and co-roots of $M$ in $G$. Define $\check{\rho} \in \frac{1}{2} \check{X}$ to be one-half the sum of the co-roots which are positive with respect to $\check{\Delta}$.

Let $\operatorname{Aut}(R)$ be the subgroup of $\operatorname{Aut}(X)$ preserving $R$ and let $\operatorname{Aut}(R, \Delta)$ be the subgroup of $\operatorname{Aut}(R)$ preserving $\Delta$. Via the action of $W$ on $R$ we may identify

$$
\operatorname{Aut}(R)=W \rtimes \operatorname{Aut}(R, \Delta) .
$$

The action of $\Gamma_{k}:=\operatorname{Gal}(\bar{k} / k)$ on $G(\bar{k})$ gives a Galois action on $X$, via the homomorphism $\varrho: \Gamma_{k} \rightarrow \operatorname{Aut}(R)$ given by $\varrho_{\gamma}(\chi)(s)=\gamma\left(\chi\left(s^{\gamma}\right)\right)$, for $\gamma \in \Gamma_{k}, \chi \in X$, and $s \in M$. This map factors through $\operatorname{Gal}\left(E_{0} / k\right)$ and the image of the cyclic subgroup $\operatorname{Gal}\left(E_{0} / K\right)=\left\langle\sigma_{0}\right\rangle$ is generated by the automorphism

$$
\vartheta:=\varrho\left(\sigma_{0}\right) \in \operatorname{Aut}(R, \Delta)
$$

of order $e$. The full image $\varrho\left(\Gamma_{k}\right)$ is generated by $\vartheta$ and the image $\phi=\varrho(F)$ of Frobenius, with the relation

$$
\phi \vartheta \phi^{-1}=\vartheta^{q} .
$$

This Frobenius element $\phi$ need not preserve $\Delta$ (it does so if $G$ is quasi-split over $k$ and $B$ is chosen to be defined over $k$ ); in general, we have a unique decomposition

$$
\phi=w \cdot \phi_{0},
$$

where $w \in W$ and $\phi_{0} \in \operatorname{Aut}(R, \Delta)$.

Since $G$ splits over $E_{0}$, we may choose a pinning on $G \times_{k} E_{0}$. This consists of root group homomorphisms $u_{\alpha}$, for each $\alpha \in R$, from the additive group to $B$ such that $u_{\alpha}$ is defined over $E_{0}$, and we have

(i) $t u_{\alpha}(b) t^{-1}=u_{\alpha}(\alpha(t) b)$ for all $t \in M\left(E_{0}\right)$ and

(ii) $\sigma_{0}\left(u_{\alpha}(b)\right)=u_{\vartheta \alpha}\left(c_{\alpha} \sigma_{0}(b)\right)$, where $c_{\alpha}= \pm 1, c_{\alpha} c_{-\alpha}=1$ and if $\alpha \in \pm \Delta$, then $c_{\alpha}=1$.

The elements

$$
n_{\alpha}:=u_{\alpha}(-1) \cdot u_{-\alpha}(1) \cdot u_{\alpha}(-1),
$$

for $\alpha \in R$, belong to $N\left(E_{0}\right)$ and generate a finite subgroup $N_{0}$ of $N\left(E_{0}\right)$. Under the projection $N \rightarrow W$, each element $n_{\alpha}$ maps to the corresponding reflection in $W$. It follows that $N\left(E_{0}\right)=N_{0} \cdot M\left(E_{0}\right)$. From (ii) above it follows that $\sigma_{0}\left(n_{\alpha}\right)=n_{\vartheta \alpha}^{ \pm 1}$ for all $\alpha \in R$, so we have

$$
\sigma_{0}\left(N_{0}\right)=N_{0}
$$


3.2. Apartments and affine root systems. Let $\mathcal{A}(S, k), \mathcal{A}(T, K), \mathcal{A}\left(M, E_{0}\right)$ be the respective apartments of the tori $S, T, M$ in the Bruhat-Tits buildings of $G(k)$, $G(K)$, and $G\left(E_{0}\right)$. Recall that $\mathcal{A}\left(M, E_{0}\right)$ is an affine space under the vector space $V:=\mathbb{R} \otimes \check{X}$, on which the Galois group $\operatorname{Gal}\left(E_{0} / k\right)$ acts by affine transformations, and we have

$$
\mathcal{A}(T, K)=\mathcal{A}\left(M, E_{0}\right)^{\operatorname{Gal}\left(E_{0} / K\right)}, \quad \mathcal{A}(S, k)=\mathcal{A}\left(M, E_{0}\right)^{\operatorname{Gal}\left(E_{0} / k\right)} .
$$

The group $N\left(E_{0}\right)$ also acts on $\mathcal{A}\left(M, E_{0}\right)$ via affine transformations. Since $N\left(E_{0}\right)=$ $N_{0} \cdot M\left(E_{0}\right)$ and $N_{0}$ is finite, it follows that $N_{0}$ has a unique fixed point $x_{0} \in$ $\mathcal{A}\left(M, E_{0}\right)$. Any linear functional $\lambda$ on $V$ then gives an affine linear function $\tilde{\lambda}$ : $\mathcal{A}\left(M, E_{0}\right) \rightarrow \mathbb{R}$, defined by $\tilde{\lambda}(x)=\lambda\left(x-x_{0}\right)$, and the gradient of $\tilde{\lambda}$ is $\lambda$. In particular, each root $\alpha \in R$ gives an affine function $\tilde{\alpha}$ on $\mathcal{A}\left(M, E_{0}\right)$.

From (3.2) it follows that $x_{0} \in \mathcal{A}(T, K)$. The system $\Psi_{K}$ of affine $K$-roots of $G(K)$ (with respect to $T$ ) may be constructed from the data $\left(R, \vartheta, x_{0}\right)$ as in 32 : Let $R / \vartheta$ be the set of orbits in $R$ under $\langle\vartheta\rangle$, and for each orbit $a \in R / \vartheta$, let $\beta_{a}$ denote the restriction to $\check{V}^{\vartheta}$ of any $\alpha \in a$. The collection

$$
R_{\vartheta}:=\left\{\beta_{a}: a \in R / \vartheta\right\}
$$

is a root system (possibly nonreduced) of linear functionals on $\check{V}^{\vartheta}$, with basis

$$
\Delta_{\vartheta}:=\left\{\beta_{a}: a \in \Delta / \vartheta\right\}
$$

It will be convenient to choose a numbering:

$$
\Delta_{\vartheta}=\left\{\beta_{1}, \ldots, \beta_{\ell_{\vartheta}}\right\}
$$

where $\ell_{\vartheta}=|\Delta / \vartheta|$ is the number of $\langle\vartheta\rangle$-orbits in $\Delta$.

There is a unique alcove $\mathcal{C} \subset \mathcal{A}(T, K)$ containing $x_{0}$ in its closure, on which $\tilde{\beta}_{i}>0$ for all $i=1, \ldots, \ell_{\vartheta}$. We define a certain negative root $\beta_{0} \in R_{\vartheta}$ as follows (see [30, 3.11]). If $\vartheta=1$, then $\beta_{0}$ is the lowest root of $R=R_{\vartheta}$. If $\vartheta \neq 1$, then $\beta_{0}$ is the lowest short root of $R_{\vartheta}$ except if $(R, \vartheta)$ has type ${ }^{2} A_{2 n}$, in which case $\beta_{0}$ is twice the lowest short root of $R_{\vartheta}$. The integers $b_{0}(=1), b_{1}, \ldots, b_{\ell_{\vartheta}}$ mentioned earlier in (2.10) are uniquely defined by the relation $\sum_{i=0}^{\ell_{\vartheta}} b_{i} \beta_{i}=0$, and the affine roots $\psi_{i}$ defining the walls of $\mathcal{C}$ are given by $\psi_{0}=\frac{1}{e}+\tilde{\beta}_{0}$ and $\psi_{i}=\tilde{\beta}_{i}$ for $i=1, \ldots, \ell_{\vartheta}$. Finally, we have

$$
\Psi_{K}=\bigsqcup_{a \in R / \vartheta} \Psi_{K}(a)
$$

where

$$
\Psi_{K}(a)= \begin{cases}\left\{\widetilde{\beta}_{a}+\frac{n}{|a|}: n \in \mathbb{Z}\right\} & \text { if } \quad \beta_{a} \notin 2 R_{\vartheta}, \\ \left\{\widetilde{\beta}_{a}+\frac{1}{2}+n: n \in \mathbb{Z}\right\} & \text { if } \quad \beta_{a} \in 2 R_{\vartheta} .\end{cases}
$$

Note that the second case only occurs in type ${ }^{2} A_{2 n}$, where $a=\{\alpha+\vartheta \alpha\}$ and $\{\alpha, \vartheta \alpha\}$ generates a subsystem of type $A_{2}$.

Let $W_{\text {aff }}(R, \vartheta)$ be the affine Weyl group of the affine root system $\Psi_{K}$. This is the group of affine transformations of $\mathcal{A}(T, K)$ generated by the reflections in the hyperplanes $\psi^{-1}(0)$ for $\psi \in \Psi_{K}$. 
3.3. Kac coordinates and barycenters. A point $x \in \mathcal{A}(T, K)$ is rational if $\psi(x) \in \mathbb{Q}$ for all affine $K$-roots $\psi \in \Psi_{K}$. When this holds, we define the order of $x$ to be the smallest positive integer $m$ such that $\psi(x) \in \frac{1}{m} \mathbb{Z}$ for all $\psi \in \Psi_{K}$.

The order is invariant under the affine Weyl group $W_{\text {aff }}(R, \vartheta)$. If $x$ is a rational point of order $m$ and $x^{\prime} \in W_{\text {aff }}(R, \vartheta) \cdot x$ is contained in the closure of the alcove $\mathcal{C}$, then there are relatively prime nonnegative integers $s_{i}$, the Kac coordinates of $x$, such that $\psi_{i}\left(x^{\prime}\right)=s_{i} / m$ for $i \in\left[0, \ell_{\vartheta}\right]$. The Kac-coordinates and the order are related by

$$
e \cdot \sum_{i=0}^{\ell_{\vartheta}} b_{i} s_{i}=m .
$$

In particular, the order $m$ is divisible by $e$.

A point $x \in \mathcal{A}(T, K)$ is a barycenter if $x$ is a rational point with all Kaccoordinates $s_{i} \in\{0,1\}$. The order $m$ of a barycenter $x$ is given by

$$
m=e \cdot \sum_{s_{i}=1} b_{i}
$$

In particular, the order of a barycenter is bounded by the twisted Coxeter number:

$$
m \leq e \cdot\left(b_{0}+b_{1}+\cdots+b_{\ell_{\vartheta}}\right)=h_{\vartheta} .
$$

Each facet $\mathcal{F}$ in the closure of $\mathcal{C}$ contains a unique barycenter, namely the point $x \in \mathcal{F}$ where all $\psi_{i}$ not vanishing on $\mathcal{F}$ take the same value. This value is $1 / m$, where $m$ is the order of $x$.

Given a point $x \in \mathcal{A}(T, K)$ and a real number $r \geq 0$, let

$$
R_{x, r}=\left\{\dot{\psi}: \psi \in \Psi_{K} \text { and } \psi(x)=r\right\} .
$$

The roots in $R_{x, r}$ are the nonzero weights of $\mathrm{V}_{x, r}$ under the torus

$$
\mathrm{T}:=T(K)_{0} / T(K)_{0+} .
$$

One checks that for $w \in W_{\text {aff }}(R, \vartheta)$ we have

$$
R_{w x, r}=\dot{w}\left(R_{x, r}\right) .
$$

Lemma 3.1. Suppose $x \in \mathcal{A}(T, K)$ is a rational point of order $m$. If $x$ is not a barycenter, then there exists a co-weight $\check{\lambda} \in \check{X}^{\vartheta}$ such that $\langle\beta, \check{\lambda}\rangle>0$ for all $\beta \in R_{x, 1 / m}$.

Proof. By (3.4) we may assume $x \in \overline{\mathcal{C}}$. If $R_{x, 1 / m}$ contains only positive or only negative roots with respect to $\Delta_{\vartheta}$, then one of $\pm 2 \check{\rho}$ satisfies the conclusion of the lemma. Hence we may also assume that $R_{x, 1 / m}$ contains a positive root as well as a negative root. by

Let $\left(s_{0}, \ldots, s_{\ell_{\vartheta}}\right)$ be the Kac coordinates of $x$, and define a function $H: R_{\vartheta} \rightarrow \mathbb{Z}$

$$
H(\beta):=m \cdot \tilde{\beta}(x)=\sum_{i=1}^{\ell_{\vartheta}} c_{i} s_{i},
$$

where $c_{i}$ is the coefficient of the simple $\operatorname{root} \beta_{i}$ in $\beta$.

Recall that $\beta=\beta_{a}$ for some $a \in R / \vartheta$ and that $|a|$ divides $e$ which divides $m$. We define positive integers $e^{\prime}, m^{\prime}$ as follows.

If $\beta \notin 2 R_{\vartheta}$, we set

$$
e^{\prime}=\frac{e}{|a|}, \quad m^{\prime}=\frac{m}{|a|},
$$


so that $e^{\prime}=1$ if $\beta$ is a short root in $R_{\vartheta}$ and $e^{\prime}=e$ if $\beta$ is long. If $\beta \in 2 R_{\vartheta}$, we set

$$
e^{\prime}=\frac{e}{2}=1, \quad m^{\prime}=\frac{m}{2} .
$$

If $m^{\prime}=1$, then $e=m$, so $x$ is a barycenter, by the linear relation (2.9), so we assume $m^{\prime}>1$.

The coefficients $c_{i}$ of $\beta$ satisfy

$$
-e^{\prime} b_{i} \leq c_{i} \leq e^{\prime} b_{i}
$$

for $i=1, \ldots, \ell_{\vartheta}$, and since $x$ has order $m$, it follows that

$$
m^{\prime}=e^{\prime}\left(s_{0}-H\left(\beta_{0}\right)\right),
$$

so we have the inequalities

$$
e^{\prime} s_{0}-m^{\prime}=e^{\prime} H\left(\beta_{0}\right) \leq H(\beta) \leq-e^{\prime} H\left(\beta_{0}\right)=m^{\prime}-e^{\prime} s_{0} .
$$

Let $\psi \in \Psi_{K}$ be an affine root with $\dot{\psi}=\beta$ and $\psi(x)=1 / m$. There is an integer $n$ such that

$$
\psi= \begin{cases}\tilde{\beta}+\frac{n}{|a|} & \text { if } \beta=\beta_{a} \notin 2 R, \\ \tilde{\beta}+\frac{n}{2} & \text { if } \beta \in 2 R .\end{cases}
$$

The equation $\psi(x)=1 / m$ is equivalent to the equation

$$
H(\beta)=1-n m^{\prime},
$$

so that the inequalities $(3.6)$ become

$$
e^{\prime} s_{0}-m^{\prime} \leq 1-n m^{\prime} \leq m^{\prime}-e^{\prime} s_{0} .
$$

Assume that $\beta$ is a negative root with respect to $\Delta_{\vartheta}$. Then $n \geq 1$, and the left-hand inequality of (3.8) yields

$$
0 \leq(n-1) m^{\prime} \leq 1-e^{\prime} s_{0} \leq 1 .
$$

But $m^{\prime}>1$ so we must have $n=1$. It also follows (since we have assumed $R_{\vartheta}$ contains negative roots) that $s_{0} \in\{0,1\}$. We now have

$$
H(\beta)=1-m^{\prime}=1-e^{\prime}\left(s_{0}-H\left(\beta_{0}\right)\right),
$$

which is equivalent to

$$
\sum_{i=1}^{\ell_{\vartheta}}\left(c_{i}+e^{\prime} b_{i}\right) s_{i}=1-e^{\prime} s_{0} .
$$

But all $c_{i}+e^{\prime} b_{i} \geq 0$, by (3.5), so we must have $c_{i}+e^{\prime} b_{i}=0$ whenever $s_{i} \geq 2$. It follows that

$$
\beta=e^{\prime} \beta_{0}+\sum_{i \in J}\left(c_{i}+e^{\prime} b_{i}\right) \beta_{i},
$$

where $J=\left\{i \in\left[1, \ell_{\vartheta}\right]: s_{i} \leq 1\right\}$.

Assume that $\beta$ is a positive root. Since $H(\beta) \geq 0$, we must have $n=0$ in equation (3.7), so $H(\beta)=1$. This means that $c_{i}=0$ whenever $s_{i} \geq 2$ and we have

$$
\beta=\sum_{i \in J} c_{i} \beta_{i} .
$$

We have shown that every root in $R_{x, 1 / m}$ is a linear combination, with nonnegative coefficients, of roots in the set $S:=\left\{\beta_{j}: j \in J \cup\{0\}\right\}$. 
The assumption of the lemma is that $J \cup\{0\} \neq\left[0, \ell_{\vartheta}\right]$. Hence $S$ is a basis of a root subsystem $R^{\prime}$ of $R_{\vartheta}$. The sum $\check{\lambda}$ of the co-roots of $R^{\prime}$ which are positive with respect to $S$ satisfies the conclusion of the lemma.

Lemma 3.1 implies that if $x$ is not a barycenter, then $\mathrm{V}_{x, 1 / m}$ and its dual space $\check{\mathrm{V}}_{x, 1 / m}$ have no nonzero closed orbits under $\mathrm{G}_{x}$. In view of (3.3), this has the following implication.

Proposition 3.2. If $x \in \mathcal{A}(T, K)$ is a rational point of order $m$ and $\check{\mathrm{V}}_{x, 1 / m}$ contains a nonzero closed orbit under $\mathrm{G}_{x}$, then $x$ is a barycenter and $m \leq h_{\vartheta}$, with equality if and only if $x$ is a barycenter of an alcove.

\section{Filtration Groups AND Graded Lie Algebras}

In this section we show that the representations $\left(\mathrm{G}_{x}, \check{\mathrm{V}}_{x, r}\right)$ arising from MoyPrasad filtrations are the same representations arising in the Vinberg-Levy theory of graded Lie algebras. This will lead to the classification of the points $x$ for which $\mathrm{G}_{x}$ has stable orbits in $\check{\mathrm{V}}_{x, 1 / m}$. We will show that such points $x$ are those barycenters which arise from $W$-conjugacy classes of elliptic $\mathbb{Z}$-regular automorphisms of the absolute root system $R$. These results require additional assumptions on $p$.

4.1. Moy-Prasad filtrations. Fix a positive integer $m$ which is divisible by $e$ and not divisible by $p$. Let $E / K$ be the unique extension of $K$ (in $\bar{k}$ ) of degree $m=[E: K]$. Then $E / K$ is totally and tamely ramified, $E$ contains the splitting field $E_{0}$ of $G$ over $K$, and $E$ is Galois over $k$. Extend the valuation $\vee$ to $E$, so that $\mathrm{v}\left(E^{\times}\right)=\frac{1}{m} \mathbb{Z}$. Let $A_{E}=\{a \in E: \mathrm{v}(a) \geq 0\}$ and $P_{E}=\{a \in E: \mathrm{v}(a)>0\}$ denote the ring of integers and prime ideal of $E$, respectively. Since $E / K$ is totally ramified, we may and shall identify $\mathfrak{F}$ with the residue field of $E$, via the inclusion $A_{K} \hookrightarrow A_{E}$.

Fix a generator $\sigma \in \operatorname{Gal}(E / K)$ (of order $m$ ) such that $\sigma=\sigma_{0}$ on $E_{0}$. We may and shall choose a uniformizer $\pi \in E$ such that $\pi^{m}=\varpi$ is a uniformizer in $k$. We have $\sigma(\pi)=\zeta \pi$, where $\zeta \in A_{K}^{\times}$is a root of unity of order $m$. Let $F$ now denote the unique Frobenius element of $\operatorname{Gal}(E / k)$ such that $F(\pi)=\pi$. Let $\vartheta=\varrho(\sigma) \in \operatorname{Aut}(R, \Delta)$ be the image of $\sigma$ under the Galois action $\varrho: \operatorname{Gal}(E / K) \rightarrow \operatorname{Aut}(R, \Delta)$ arising from the $K$-structure on $G \times{ }_{k} K$.

We have $\mathcal{A}(M, E)=\mathcal{A}\left(M, E_{0}\right)$ and the set of affine functions

$$
\Psi_{E}:=\left\{\tilde{\alpha}+\frac{n}{m}: \alpha \in R, n \in \mathbb{Z}\right\}
$$

is the system of affine $E$-roots of $G(E)$ (with respect to $M(E)$ ), and $x_{0}$ is a hyperspecial point in $\mathcal{A}(M, E)$.

Each affine $E$-root $\psi \in \Psi_{E}$ with gradient $\dot{\psi}$ determines an affine root group $U_{\psi} \subset G(E)$, which may be described explicitly in terms of the basepoint $x_{0}$ and the root groups $\left\{u_{\alpha}: \alpha \in R\right\}$ as

$$
U_{\psi}=\left\{u_{\dot{\psi}}(b): b \in E, \mathbf{v}(b) \geq \psi\left(x_{0}\right)\right\},
$$

and for each $x \in \mathcal{A}(M, E)$ and $r \geq 0$ the Moy-Prasad filtration subgroup of $G(E)$ is given by

$$
G(E)_{x, r}=\left\langle U_{\psi}, M(E)_{r}: \psi \in \Psi_{E}, \psi(x) \geq r\right\rangle
$$

where $M(E)_{0}=\check{X} \otimes A_{E}^{\times}$is the parahoric subgroup of $M(E)$ and

$$
M(E)_{r}=\left\{t \in M(E)_{0}: \mathbf{v}(\chi(t)-1) \geq r \quad \forall \chi \in X\right\},
$$


for $r>0$. As before, we set

$$
G(E)_{x, r+}=\bigcup_{s>r} G(E)_{x, s}, \quad \overline{G(E)}_{x, r}=G(E)_{x, r} / G(E)_{x, r+} .
$$

The group $G(E)_{x, 0}$ is the parahoric subgroup of $G(E)$ attached to the point $x$ via Bruhat-Tits theory; it is contained with finite index in the full stabilizer $G(E)_{x}$ of $x$ in $G(E)$. The quotient $\overline{G(E)}_{x, 0}$ is a connected reductive group over the residue field $\mathfrak{F}$ of $E$, and for $r>0$ the quotient $\overline{G(E)}_{x, r}$ is a finite-dimensional $\mathfrak{F}$-vector space, affording a rational representation of $\overline{G(E)}_{x, 0}$, induced by conjugation.

If $x=x_{0}$ and $r=0$, we have

$$
\begin{aligned}
G(E)_{x_{0}, 0} & =\left\langle u_{\alpha}\left(A_{E}\right), \check{\lambda}\left(A_{E}^{\times}\right): \alpha \in R, \check{\lambda} \in \check{X}\right\rangle, \\
G(E)_{x_{0}, 0+} & =\left\langle u_{\alpha}\left(P_{E}\right), \check{\lambda}\left(1+P_{E}\right): \alpha \in R, \check{\lambda} \in \check{X}\right\rangle .
\end{aligned}
$$

The quotient

$$
\mathrm{G}:=\overline{G(E)}_{x_{0}, 0}
$$

is a connected reductive $\mathfrak{f}$-group with the same based root datum $(X, \Delta, \check{X}, \check{\Delta})$ as that of $G$ and $\mathfrak{f}$-structure given by the Frobenius element $\phi_{0}$ (see (3.1)).

The Galois action of $\operatorname{Gal}(E / K)=\langle\sigma\rangle$ on $G(E)$ imparts additional structure to $\mathrm{G}$. For each $\alpha \in R$ let $\mathrm{u}_{\alpha}: \mathfrak{F}^{+} \rightarrow \mathrm{G}$ be the root group fitting into the commutative diagram

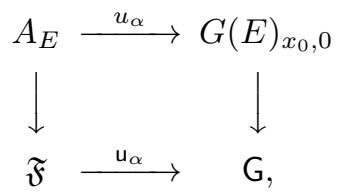

where the vertical maps are the obvious projections. There is a unique algebraic automorphism of $\mathrm{G}$, again denoted by $\vartheta$, such that $\vartheta\left(\mathbf{u}_{\alpha}(t)\right)=\mathbf{u}_{\vartheta(\alpha)}\left(c_{\alpha} t\right)$ for each $\alpha \in R$ and $t \in \mathfrak{F}$, where $c_{\alpha}= \pm 1$ and $c_{\alpha}=+1$ if $\alpha \in \pm \Delta$. Since $E / K$ is totally ramified, it follows that $\mathrm{u}_{\alpha}(\mathfrak{F})$ is in fact the projection to $\mathrm{G}$ of $u_{\alpha}\left(A_{K}\right)$, which implies that $\sigma\left(\mathbf{u}_{\alpha}(t)\right)=\vartheta\left(\mathbf{u}_{\alpha}(t)\right)$ for all $t \in \mathfrak{F}^{\times}$. Likewise $\check{\lambda}\left(A_{E}^{\times}\right)$and $\check{\lambda}\left(A_{K}^{\times}\right)$have the same projection to $\mathrm{G}$, for each $\check{\lambda} \in \check{X}$, so $\sigma(\check{\lambda}(t))=\vartheta(\check{\lambda}(t))$ for $t \in \mathfrak{F}^{\times}$. Thus, the Galois action of $\sigma$ on $G(E)_{x_{0}, 0}$ induces the algebraic action of $\vartheta$ on $\mathrm{G}$, and we have

$$
\left(\mathrm{G}^{\vartheta}\right)^{\circ}=\overline{G(K)}_{x_{0}, 0} .
$$

Let $\mathfrak{L}$ be the Lie algebra of $G$. As before, we may identify

$$
\mathfrak{L}=\overline{\mathfrak{g}(E)}_{x_{0}, 0}
$$

and the Galois action of $\sigma$ on $\mathfrak{g}(E)_{x_{0}, 0}$ induces the algebraic action of $\vartheta$ on $\mathfrak{L}$, so that

$$
\mathfrak{L}^{\vartheta}=\overline{\mathfrak{g}(K)}_{x_{0}, 0} .
$$

If $x \in \mathcal{A}(T, K)$, then $\sigma(x)=x$ and it is clear that we have equality of full stabilizers:

$$
\left(G(E)_{x}\right)^{\sigma}=G(K)_{x}
$$

and since $E / K$ is tame, we also have, for $r>0$,

$$
\left(G(E)_{x, r}\right)^{\sigma}=G(K)_{x, r} .
$$


When $r=0$, equality (4.3) need not hold, because the group $\left(\overline{G(E)}_{x, 0}\right)^{\sigma}$ need not be connected; its identity component is $\overline{G(K)}_{x, 0}$.

4.2. Graded Lie algebras. We are ready to show that the representation pairs $\left(\mathrm{G}_{x}, \mathrm{~V}_{x, r}\right)$ are exactly those appearing in the Vinberg-Levy theory of graded Lie algebras.

Let $x \in \mathcal{A}(S, k)$ be a rational point of order $m$. Then $x$ becomes hyperspecial in $\mathcal{A}(M, E)$. The Lie algebra $\mathfrak{g}(E)$ is filtered by Moy-Prasad lattices $\mathfrak{g}(E)_{x, r}$, with jumps in $\frac{1}{m} \mathbb{Z}$, and we have $\mathfrak{g}(E)_{x, i / m}=\pi^{i} \mathfrak{g}(E)_{x, 0}$ for all $i>0$, along with canonical isomorphisms on successive quotients

$$
{\overline{G(E)_{x, i / m}}}=G(E)_{x, i / m} / G(E)_{x,(i+1) / m} \simeq \mathfrak{g}(E)_{x, i / m} / \mathfrak{g}(E)_{x,(i+1) / m}={\overline{\mathfrak{g}(E)_{x, i / m}}}
$$

which are equivariant for the $\operatorname{Gal}(E / k)$-action.

This action of $\operatorname{Gal}(E / k)$ depends on $x$. To see this explicitly, write

$$
x=x_{0}-\frac{1}{m} \check{\lambda},
$$

where $\check{\lambda} \in \check{X}^{\vartheta}$. Consider the elements in $M(E)$ given by

$$
t:=\check{\lambda}(\pi) \quad \text { and } \quad s=\check{\lambda}(\zeta),
$$

which satisfy $\sigma(t)=t s$. Identifying $\zeta$ with its image in $\mathfrak{F}^{\times}$, we may regard $s$ as an element of $\mathrm{T}$, so that $\operatorname{Ad}(s)$ is an inner automorphism of $\mathfrak{L}$. We set

$$
\theta:=\operatorname{Ad}(s) \vartheta \in \operatorname{Aut}(\mathfrak{L}) .
$$

Under the translation action of $M(E)$ on $\mathcal{A}(M, E)$ (see [36, 1.2]), we have $t \cdot x_{0}=x$, so that the conjugation map $c_{t}: g \mapsto t g t^{-1}$ sends $G(E)_{x_{0}, 0}$ to $G(E)_{x, 0}$ and thereby induces an $\mathfrak{F}$-linear isomorphism

$$
f: \mathrm{G} \stackrel{\sim}{\longrightarrow} \overline{G(E)}_{x, 0} .
$$

Since $\sigma(t)=t s$, it follows that $c_{t s} \circ \sigma=\sigma \circ c_{t}$. But we have seen that $\sigma$ induces $\vartheta$ on $\mathrm{G}$. It follows that the isomorphism $f$ has the intertwining property:

$$
f \circ \theta=\sigma \circ f,
$$

so

$$
f\left(\mathrm{G}^{\theta}\right)=\left({\overline{G(E)_{x, 0}}}\right)^{\sigma} .
$$

Letting $f_{0}$ denote the restriction of $f$ to $\left(\mathrm{G}^{\theta}\right)^{\circ}$, we get an isomorphism on identity components:

$$
f_{0}:\left(\mathrm{G}^{\theta}\right)^{\circ} \stackrel{\sim}{\longrightarrow} \mathrm{G}_{x}={\overline{G(K)_{x, 0}}} .
$$

Recall that we have taken $x \in \mathcal{A}(S, k)$, so that $F(x)=x$ and $F$ preserves $G(K)_{x, 0}$, inducing an $\mathfrak{f}$-structure on the algebraic group $\mathrm{G}_{x}$. We need not have $F\left(x_{0}\right)=x_{0}$, so $F$ does not act on $G$. However, the isomorphism (4.6) does descend to $\mathfrak{f}$, provided we give $\mathrm{G}$ the $\mathfrak{f}$-structure with Frobenius

$$
F_{f}:=f^{-1} \circ F \circ f \text {. }
$$

Turning to higher depths, for each $1 \leq i<m$, the eigenspace

$$
\mathfrak{L}\left(\theta, \zeta^{-i}\right):=\left\{v \in \mathfrak{L}: \theta(v)=\zeta^{-i} v\right\}
$$


affords a representation of $\mathrm{G}^{\theta}$. We define an $\mathfrak{F}$-linear isomorphism

$$
f_{i}: \mathfrak{L}\left(\theta, \zeta^{-i}\right) \longrightarrow \mathrm{V}_{x, i / m}
$$

as follows.

Let $v \in \mathfrak{L}\left(\theta, \zeta^{-i}\right)$. Recalling that $f$ is induced by $c_{t}$, we have from (4.5) that

$$
\sigma(d f(v))=\zeta^{-i} \cdot d f(v),
$$

where $d f: \mathfrak{L} \rightarrow \overline{\mathfrak{g}(E)}_{x, 0}$ is the differential of $f$.

Let $\tilde{v} \in \mathfrak{g}(E)_{x_{0}, 0}$ be a lift of $v$. Then $\operatorname{Ad}(t) \tilde{v} \in \mathfrak{g}(E)_{x, 0}$ is a lift of $d f(v)$, so equation (4.8) implies that

$$
\sigma(\operatorname{Ad}(t) \tilde{v}) \equiv \zeta^{-i} \operatorname{Ad}(t) \tilde{v} \quad \bmod \mathfrak{g}(E)_{x, 1 / m} .
$$

Since $\sigma\left(\pi^{i}\right)=\zeta^{i} \pi^{i}$ and $\pi^{i} \operatorname{Ad}(t) \tilde{v} \in \mathfrak{g}(E)_{x, i / m}$, it follows from (4.9) that

$$
\sigma\left(\pi^{i} \operatorname{Ad}(t) \tilde{v}\right) \equiv \pi^{i} \operatorname{Ad}(t) \tilde{v} \quad \bmod \mathfrak{g}(E)_{x,(i+1) / m} .
$$

Therefore, $\pi^{i} \operatorname{Ad}(t) \tilde{v}$ projects to a $\sigma$-fixed element

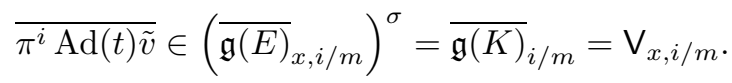

One checks that the map

$$
f_{i}: \mathfrak{L}\left(\theta, \zeta^{-i}\right) \longrightarrow \mathrm{V}_{x, i / m}
$$

given by

$$
f_{i}(v)=\overline{\pi^{i} \operatorname{Ad}(t) \tilde{v}}
$$

is a well-defined isomorphism intertwining the adjoint action of an element $g \in\left(\mathrm{G}^{\theta}\right)^{\circ}$ on $\mathfrak{L}\left(\theta, \zeta^{-i}\right)$ with the conjugation action of $f_{0}(g) \in \mathrm{G}_{x}$ on $\mathrm{V}_{x, i / m}$.

Assume there is a nondegenerate G-invariant symmetric pairing $\mathfrak{L} \times \mathfrak{L} \rightarrow \mathfrak{F}$. (This holds, for example, if $p$ is odd and is not a torsion prime for G.) Then $\mathfrak{L}\left(\theta, \zeta^{i}\right)$ and $\mathfrak{L}\left(\theta, \zeta^{-i}\right)$ become contragredient representations of $\left(\mathrm{G}^{\theta}\right)^{\circ}$. Thus $f_{i}$ induces an isomorphism

$$
\check{f}_{i}: \mathfrak{L}\left(\theta, \zeta^{i}\right) \stackrel{\sim}{\longrightarrow} \check{V}_{x, i / m} .
$$

We summarize what we have shown in this section:

Theorem 4.1. Assume there is a $\mathrm{G}$-invariant nondegenerate symmetric form on $\mathfrak{L}$ and let $x \in \mathcal{A}(T, K)$ be a rational point of order $m$ such that $p \nmid m$. Let $\check{\lambda} \in \check{X}^{\vartheta}$ be defined by $x_{0}-x=\frac{1}{m} \check{\lambda}$, and let $E=K(\pi)$ be the unique tame extension $K$ of degree $m$, where $\pi$ is a uniformizer in $E$ such that $\pi^{m}$ is a uniformizer in $k$. Choose a generator $\sigma$ of $\operatorname{Gal}(E / K)$, identify the $m^{\text {th }}$ root of unity $\zeta:=\sigma(\pi) / \pi$ with its image in $\mathfrak{F}^{\times}$, and define

$$
s=\check{\lambda}(\zeta) \in \mathrm{T}, \quad \theta=\operatorname{Ad}(s) \vartheta \in \operatorname{Aut}(\mathfrak{L}),
$$

where $\vartheta=\varrho(\sigma)$. Then for $1 \leq i<m$ the isomorphisms

$$
f_{0}:\left(\mathrm{G}^{\theta}\right)^{\circ} \stackrel{\sim}{\longrightarrow} \overline{G(K)}_{x, 0}, \quad \check{f}_{i}: \mathfrak{L}\left(\theta, \zeta^{i}\right) \stackrel{\sim}{\longrightarrow} \check{\mathrm{V}}_{x, i / m},
$$

defined in (4.6) and (4.11), fit into a commutative diagram

$$
\begin{aligned}
& \left(\mathrm{G}^{\theta}\right)^{\circ} \stackrel{\mathrm{Ad}}{\longrightarrow} \mathrm{GL}\left(\mathfrak{L}\left(\theta, \zeta^{i}\right)\right) \\
& f_{0} \downarrow \simeq \quad \operatorname{Ad}\left(\check{f}_{i}\right) \downarrow \simeq \\
& \mathrm{G}_{x} \longrightarrow \mathrm{GL}\left(\check{\mathrm{V}}_{x, i / m}\right) \text {, }
\end{aligned}
$$


where the lower horizontal map is the dual of the representation of $\mathrm{G}_{x}$ on $\mathrm{V}_{x, i / m}$ induced by the conjugation action of $G(K)_{x, 0}$ on $G(K)_{x, i / m}$ and $\operatorname{Ad}\left(\check{f}_{i}\right)$ is conjugation by $\check{f}_{i}$.

Remark 1. Assuming only that $p \nmid e$, a point $x \in \mathcal{A}(S, k)$ of order $m$ still gives a $\mathbb{Z} / m$-grading $\mathfrak{L}=\oplus \mathfrak{L}_{i}$ (cf. [33, [32, 1.3]) such that the representations $\mathrm{V}_{x, i / m}$ and $\mathfrak{L}_{i}$ have the same decomposition under T. It is likely that a version of Theorem 4.1 can be proved in this more general setting.

Remark 2. Recall that the Frobenius $F$ on $G(E)_{x, 0}$ transfers to the twisted Frobenius $F_{f}$ on $G(E)_{x_{0}, 0}$, defined in (4.7). On the latter group we have the relation

$$
F_{f} \circ \theta=\theta^{q} \circ F_{f},
$$

which follows from (4.5). This implies that $F_{f}$ preserves each eigenspace $\mathfrak{L}\left(\theta, \zeta^{i}\right)$, and one checks that the vertical maps in the diagram of Theorem 4.1 interwine the action of $F_{f}$ on the top row with $F$ on the bottom row.

\section{Stable orbits And ePiPELAGiC REPRESENTAtions}

In this section we assume that $p$ is odd and is not a torsion prime for $G$, and we keep the notation of Section 4.2. Let $x \in \mathcal{A}(T, K)$ be a rational point of order $m$, where $p \nmid m$. By Theorem 4.1, the group $\mathrm{G}_{x}$ has stable orbits in $\check{\mathrm{V}}_{x, 1 / m}$ if and only if the group $\mathrm{G}_{0}:=\left(\mathrm{G}^{\theta}\right)^{\circ}$ has stable orbits in the eigenspace $\mathrm{V}:=\mathfrak{L}(\theta, \zeta)$.

The latter eigenspace representations were analyzed by Vinberg [37] (over $\mathbb{C}$ ) and his results were extended by Levy 24] to algebraically closed fields of good odd positive characteristic. The main features of this theory are as follows. Define a Cartan subspace to be a subspace of $\mathrm{V}$ which is abelian under the Lie bracket in $\mathfrak{L}$, consists entirely of semisimple elements of $\mathfrak{L}$, and is maximal with these two properties. Then: (1) Any two Cartan subspaces are conjugate under $\mathrm{G}_{0}$, every closed $\mathrm{G}_{0}$-orbit in $\mathrm{V}$ meets a fixed Cartan subspace $\mathfrak{c}$, and if two points in $\mathfrak{c}$ are $\mathrm{G}_{0}$-conjugate, then they are conjugate under the normalizer $N_{\mathrm{G}_{0}}(\mathfrak{c})$. (2) The action of the faithful quotient $W(\mathfrak{c}, \theta)=N_{\mathrm{G}_{0}}(\mathfrak{c}) / C_{\mathrm{G}_{0}}(\mathfrak{c})$ on $\mathfrak{c}$ is generated by reflections. (3) The restriction map gives an isomorphism on rings of polynomial invariants:

$$
\mathfrak{F}[\mathrm{V}]^{\mathrm{G}_{0}} \stackrel{\sim}{\longrightarrow} \mathfrak{F}[\mathfrak{c}]^{W(\mathfrak{c}, \theta)},
$$

and these rings are generated by homogeneous polynomials $f_{1}, \ldots, f_{r}$, where $r=$ $\operatorname{dim} \mathfrak{c}$ is the rank of $\mathrm{V}$, and we have $\prod_{i=1}^{r} \operatorname{deg}\left(f_{i}\right)=|W(\mathfrak{c}, \theta)|$.

If the rank of $\mathrm{V}$ is zero (the unstable case), then every orbit of $\mathrm{G}_{0}$ in $\mathrm{V}$ is nilpotent and $\{0\}$ is the only closed orbit. So $G_{0}$ has semistable orbits in $V$ exactly when $V$ has positive rank. The pairs $\left(\mathrm{G}_{0}, \mathrm{~V}\right)$ of positive rank were classified in [37, 24], and [32]; we shall discuss this further in Section 8 .

The subset of stable representations $\left(G_{0}, V\right)$ (i.e., those having closed orbits with finite isotropy groups) was also classified in 32 . These are in bijection with certain $W$-conjugacy classes of automorphisms of $R$, as we now explain.

We say that an automorphism $\sigma \in \operatorname{Aut}(R)$ is $\mathbb{Z}$-regular if the group generated by $\sigma$ acts freely on $R 2$ We also say that $\sigma$ is elliptic if $\sigma$ fixes no nonzero vector in $V$. The $\mathbb{Z}$-regular elliptic elements are tabulated for exceptional groups in Section 5.1 (see also [32, Section 7]).

\footnotetext{
${ }^{2}$ The $\mathbb{Z}$-regular elements of order $m$ are those which are regular in the sense of Springer 34 and have a regular eigenvalue of order $m$.
} 
By [32, Cor. 14], the eigenspace representation $\mathrm{V}=\mathfrak{L}(\theta, \zeta)$ contains a stable orbit under $G_{0}$ precisely when $\theta$ normalizes a Cartan subalgebra $\mathfrak{s}$ of $\mathfrak{L}$ such that the restriction $\left.\theta\right|_{\mathfrak{s}}$ gives an elliptic $\mathbb{Z}$-regular $W$-conjugacy class in $\operatorname{Aut}(R)$. In this case, $\theta$ is G-conjugate to $\operatorname{Ad}(\check{\rho}(\zeta)) \vartheta$, where $\vartheta$ is the projection of $\left.\theta\right|_{\mathfrak{s}}$ to $\operatorname{Aut}(R, \Delta)$. Moreover, this is independent of the choice of $\zeta$, in the sense that if $\zeta^{\prime}$ is another root of unity of order $m$, then the automorphisms $\operatorname{Ad}(\check{\rho}(\zeta)) \vartheta$ and $\operatorname{Ad}\left(\check{\rho}\left(\zeta^{\prime}\right)\right) \vartheta$ are G-conjugate.

Using Theorem 4.1, with $x$ and $\theta$ as related therein, we can transfer this classification to $\check{\mathrm{V}}_{x, 1 / m}$ and obtain the following.

Corollary 5.1. Let $x \in \mathcal{A}(T, K)$ be a rational point of order $m$. Then $\check{\mathbf{V}}_{x, 1 / m}$ contains stable vectors under $G_{x}$ if and only if $m$ is the order of an elliptic $\mathbb{Z}$-regular element of $w \vartheta \in W \vartheta$ and $x$ is conjugate under $W_{\mathrm{aff}}(R, \vartheta)$ to the point $x_{0}+\frac{1}{m} \check{\rho}$.

In Corollary 5.1, the $W$-conjugacy class of $w \vartheta$ is determined by $m$. All stabilizers $A_{0}$ in $G_{0}$ of stable vectors in $\mathfrak{L}(\theta, \zeta)$ are $\mathrm{G}_{0}$-conjugate and fit into an exact sequence

$$
1 \longrightarrow \mathrm{A}_{0} \longrightarrow \mathrm{S}^{\theta} \longrightarrow \mathrm{Z}_{\vartheta} \longrightarrow 1 \text {. }
$$

Here $Z_{\vartheta}=\mathbf{Z} /(1-\vartheta) Z$, where $\mathbf{Z}$ is the fundamental group of $G$ and $S^{\theta}$ is the group of $\theta$-invariants in the centralizer $S$ of $\mathfrak{c}$ in $\mathbf{G}$ (see [32, Section 6.3], where $A_{0}$ is denoted by $\left.S_{0}\right)$. The group $\mathrm{S}^{\theta}$ is finite, of order

$$
\left|\mathrm{S}^{\theta}\right|=\operatorname{det}\left(1-\left.w \vartheta\right|_{X}\right),
$$

and the exponent of $\mathrm{S}^{\theta}$ divides $m$ (see [31, 2.1]). Our assumption that $p \nmid m$ therefore implies that $\mathrm{A}_{0}$ has order prime to $p$.

The group $A_{x, \lambda}$ of Section 2.5 is isomorphic, via the map $f_{0}$ of Theorem 4.1] to the group of $\mathfrak{f}$-rational points in $\mathrm{A}_{0}$, and the group $H_{x, \lambda}$ is the pre-image of $A_{x, \lambda}$ in $G(k)_{x}$. Hence the finite group $A_{x, \lambda}$ is abelian of order prime to $p$, and from Remark 1 of Section 2.1, it follows that $\chi_{\lambda}$ extends to $H_{x, \lambda}$ and the intertwining algebra $\mathcal{H}_{x, \lambda}$ of Proposition 2.4 is just the group algebra of $A_{x, \lambda}$. We have shown the following.

Proposition 5.2. Let $m$ be the order of an elliptic $\mathbb{Z}$-regular automorphism $w \vartheta \in$ $W \vartheta$ and assume $p$ is a nontorsion prime for $G$, not dividing $2 m$. Let $x=x_{0}+\frac{1}{m} \check{\rho} \in$ $\mathcal{A}(S, k)$. Then $\check{\mathrm{V}}_{x, 1 / m}$ contains stable orbits under $\mathrm{G}_{x}$. If $\lambda \in \check{\mathrm{V}}_{x, 1 / m}(\mathfrak{f})$ is an $\mathfrak{f}$ rational stable vector, then the character $\chi_{\lambda}$ of $J_{x}=G(k)_{x, 1 / m}$ (see Section 2.5) extends to $H_{x, \lambda}$ and we have the direct sum decomposition

$$
\operatorname{ind}_{J_{x}}^{G(k)} \chi_{\lambda}=\bigoplus_{\rho \in \operatorname{Irr}\left(A_{x, \lambda}\right)} \pi_{x}(\lambda, \rho),
$$

where $\rho$ runs over the irreducible characters of the abelian group $A_{x, \lambda}$ and

$$
\pi_{x}(\lambda, \rho)=\operatorname{ind}_{H_{x, \lambda}}^{G(k)}\left(\chi_{\lambda} \otimes \rho\right)
$$

is an irreducible supercuspidal epipelagic representation of $G(k)$.

5.1. Tables for exceptional groups. In this section we tabulate the points $x \in$ $\mathcal{A}(T, K)$ for which $\mathrm{G}_{x}$ has stable orbits in $\check{\mathrm{V}}_{x, 1 / m}$. For brevity we list just the exceptional groups, by their "Name" in the left-most column of [36, p. 61], and we take them to be simply connected. The latter condition affects only the group $A_{0}$ (see (5.1)). For classical groups, see [32, 7.2]. 
$E_{6}$

\begin{tabular}{|c|c|c|c|c|}
\hline$m$ & $x$ & $w$ & $\mathrm{~A}_{0}$ & $\operatorname{dim} \mathfrak{c}$ \\
\hline $12=h_{\vartheta}$ & $\begin{array}{llll}1 & 1 & 1 & 1 \\
& & & 1 \\
& & & 1\end{array}$ & $E_{6}$ & $\boldsymbol{\mu}_{3}$ & 1 \\
\hline
\end{tabular}

$\begin{array}{lcccccccc} & 1 & 1 & 0 & 1 & 1 & & & \\ 9 & & & 1 & & & E_{6}\left(a_{1}\right) & \boldsymbol{\mu}_{3} & 1\end{array}$

$\begin{array}{ccccccccc}6 & 1 & 0 & 1 & 0 & 1 & & & \\ 6 & & & 0 & & & E_{6}\left(a_{2}\right) & \boldsymbol{\mu}_{3} & 2\end{array}$

$\begin{array}{lllllllllll}0 & 0 & 1 & 0 & 0\end{array}$

$\begin{array}{lllll}3 & 0 & 3 A_{2} & \boldsymbol{\mu}_{3}^{3} & 3\end{array}$

$F_{4}^{I}$

\begin{tabular}{ccccc}
\hline$m$ & $x$ & $w$ & $\mathrm{~A}_{0}$ & $\operatorname{dim} \mathfrak{c}$ \\
\hline $18=h_{\vartheta}$ & $111 \Leftarrow 11$ & $-E_{6}\left(a_{1}\right)$ & 1 & 1 \\
12 & $110 \Leftarrow 11$ & $-E_{6}$ & 1 & 1 \\
6 & $100 \Leftarrow 10$ & $-\left(3 A_{2}\right)$ & 1 & 3 \\
4 & $000 \Leftarrow 10$ & $-D_{4}\left(a_{1}\right)$ & $\boldsymbol{\mu}_{4} \times \boldsymbol{\mu}_{4}$ & 2 \\
2 & $000 \Leftarrow 01$ & -1 & $\boldsymbol{\mu}_{2}^{6}$ & 6 \\
\hline
\end{tabular}

$E_{7}$

\begin{tabular}{|c|c|c|c|c|c|c|c|}
\hline$m$ & & & $x$ & & $w$ & $A_{0}$ & $\operatorname{dim} \mathfrak{c}$ \\
\hline $18=h_{\vartheta}$ & 1 & 1 & $\begin{array}{ll}1 & 1 \\
& 1\end{array}$ & $\begin{array}{lll}1 & 1 & 1\end{array}$ & $E_{7}$ & $\mu_{2}$ & 1 \\
\hline 14 & 1 & 1 & $\begin{array}{rr}1 & 0 \\
& 1\end{array}$ & $\begin{array}{lll}1 & 11\end{array}$ & $E_{7}\left(a_{1}\right)$ & $\mu_{2}$ & 1 \\
\hline 6 & 1 & 0 & $\begin{array}{rr}0 & 1 \\
& 0\end{array}$ & $\begin{array}{lll}0 & 0\end{array}$ & $E_{7}\left(a_{4}\right)$ & $\mu_{2}$ & 3 \\
\hline 2 & c & 0 & $\begin{array}{ll}0 & 0 \\
& 1\end{array}$ & $\begin{array}{lll}0 & 0 & 0\end{array}$ & $7 A_{1}$ & $\boldsymbol{\mu}_{2}^{7}$ & 7 \\
\hline
\end{tabular}


$E_{8}$

\begin{tabular}{|c|c|c|c|c|c|c|}
\hline$m$ & & $x$ & & $w$ & $A_{0}$ & $\operatorname{dim} \mathfrak{c}$ \\
\hline $30=h_{\vartheta}$ & $\begin{array}{llll}1 & 1 & 1 & 1 \\
& & & 1\end{array}$ & $\begin{array}{ll}1 & 1 \\
\end{array}$ & $\begin{array}{ll}11 \\
\end{array}$ & $E_{8}$ & 1 & 1 \\
\hline 24 & $\begin{array}{cccc}1 & 1 & 0 & 1 \\
& & 1\end{array}$ & $\begin{array}{ll}1 & 1\end{array}$ & 11 & $E_{8}\left(a_{1}\right)$ & 1 & 1 \\
\hline 20 & $\begin{array}{cccc}1 & 1 & 0 & 1 \\
& & 1\end{array}$ & $\begin{array}{ll}0 & 1\end{array}$ & 11 & $E_{8}\left(a_{2}\right)$ & 1 & 1 \\
\hline 15 & $\begin{array}{cccc}1 & 0 & 1 & 0 \\
& & 0\end{array}$ & 10 & 11 & $E_{8}\left(a_{5}\right)$ & 1 & 1 \\
\hline 12 & $\begin{array}{cccc}1 & 0 & 1 & 0 \\
& & 0\end{array}$ & $\begin{array}{ll}0 & 1\end{array}$ & $\begin{array}{ll}0 & 1\end{array}$ & $E_{8}\left(a_{3}\right)$ & 1 & 2 \\
\hline 10 & $\begin{array}{llll}0 & 0 & 1 & 0 \\
& & 0\end{array}$ & $\begin{array}{ll}0 & 1\end{array}$ & $\begin{array}{ll}0 & 1\end{array}$ & $E_{8}\left(a_{6}\right)=-2 A_{4}$ & 1 & 2 \\
\hline 8 & $\begin{array}{llll}0 & 0 & 1 & 0 \\
& & 0\end{array}$ & $\begin{array}{ll}0 & 0\end{array}$ & 10 & $D_{8}\left(a_{3}\right)$ & $\boldsymbol{\mu}_{2} \times \boldsymbol{\mu}_{2}$ & 2 \\
\hline 6 & $\begin{array}{cccc}0 & 0 & 0 & 1 \\
& & 0\end{array}$ & $\begin{array}{ll}0 & 0 \\
& \end{array}$ & $\begin{array}{ll}0 & 1\end{array}$ & $E_{8}\left(a_{8}\right)=-4 A_{2}$ & 1 & 4 \\
\hline 5 & $\begin{array}{cccc}0 & 0 & 0 & 1 \\
& & 0\end{array}$ & $\begin{array}{ll}0 & 0 \\
& \end{array}$ & $\begin{array}{ll}0 & 0\end{array}$ & $2 A_{4}$ & $\boldsymbol{\mu}_{5} \times \boldsymbol{\mu}_{5}$ & 2 \\
\hline 4 & $\begin{array}{cccc}0 & 0 & 0 & 0 \\
& & 0\end{array}$ & 10 & $\begin{array}{ll}0 & 0\end{array}$ & $2 D_{4}\left(a_{1}\right)$ & $\boldsymbol{\mu}_{2}^{4}$ & 4 \\
\hline 3 & $\begin{array}{cccc}0 & 0 & 0 & 0 \\
& & 1\end{array}$ & $\begin{array}{ll}0 & 0 \\
& \end{array}$ & $\begin{array}{ll}0 & 0\end{array}$ & $4 A_{2}$ & $\mu_{3}^{4}$ & 4 \\
\hline 2 & $\begin{array}{cccc}1 & 0 & 0 & 0 \\
& & 0 & \\
\end{array}$ & $\begin{array}{ll}0 & 0 \\
\end{array}$ & $\begin{array}{ll}0 & 0 \\
\end{array}$ & $8 A_{1}=-1$ & $\boldsymbol{\mu}_{2}^{8}$ & 8 \\
\hline
\end{tabular}

$F_{4}$

\begin{tabular}{ccccc}
\hline$m$ & $x$ & $w$ & $\mathrm{~A}_{0}$ & $\operatorname{dim} \mathfrak{c}$ \\
\hline $12=h_{\vartheta}$ & $111 \Rightarrow 11$ & $F_{4}$ & 1 & 1 \\
8 & $111 \Rightarrow 01$ & $B_{4}$ & $\boldsymbol{\mu}_{2}$ & 1 \\
6 & $101 \Rightarrow 01$ & $F_{4}\left(a_{1}\right)$ & 1 & 2 \\
4 & $101 \Rightarrow 00$ & $D_{4}\left(a_{1}\right)$ & $\boldsymbol{\mu}_{2} \times \boldsymbol{\mu}_{2}$ & 2 \\
3 & $001 \Rightarrow 00$ & $A_{2}+\tilde{A}_{2}$ & $\boldsymbol{\mu}_{3} \times \boldsymbol{\mu}_{3}$ & 2 \\
2 & $010 \Rightarrow 00$ & $4 A_{1}$ & $\boldsymbol{\mu}_{2}^{4}$ & 4 \\
\hline
\end{tabular}

$G_{2}$

\begin{tabular}{ccccc}
\hline$m$ & $x$ & $w$ & $\mathrm{~A}_{0}$ & $\operatorname{dim} \mathfrak{c}$ \\
\hline $6=h_{\vartheta}$ & $11 \Rightarrow 1$ & $G_{2}$ & 1 & 1 \\
3 & $11 \Rightarrow 0$ & $A_{2}$ & $\boldsymbol{\mu}_{3}$ & 1 \\
2 & $01 \Rightarrow 0$ & $A_{1}+\tilde{A}_{1}$ & $\boldsymbol{\mu}_{2} \times \boldsymbol{\mu}_{2}$ & 2 \\
\hline
\end{tabular}




\begin{tabular}{ccccc}
\multicolumn{5}{c}{$G_{2}^{I}$} \\
\hline$m$ & $x$ & $w$ & $\mathrm{~A}_{0}$ & $\operatorname{dim} \mathfrak{c}$ \\
\hline $12=h_{\vartheta}$ & $11 \Leftarrow 1$ & $F_{4}$ & 1 & 1 \\
6 & $10 \Leftarrow 1$ & $F_{4}\left(a_{1}\right)$ & 1 & 2 \\
3 & $00 \Leftarrow 1$ & $A_{2}+\tilde{A}_{2}$ & $\boldsymbol{\mu}_{3} \times \boldsymbol{\mu}_{3}$ & 2 \\
\hline
\end{tabular}

\section{Cartan subspaces}

We have so far considered stable orbits over the algebraic closure $\mathfrak{F}$ of the residue field $\mathfrak{f}$ of $k$. We now examine how these orbits break up when we descend to $\mathfrak{f}$. In Section 7.3 we will use this analysis to attach Langlands parameters to epipelagic representations.

Since each stable orbit determines a Cartan subspace, we must first analyze the $\mathfrak{f}$-rational conjugacy classes of Cartan subspaces. As this is purely a question about groups over $\mathfrak{f}$, we restrict ourselves to that context.

6.1. Rational classes of Cartan subspaces. Let $G$ be a connected reductive group over a finite field $\mathfrak{f} \simeq \mathbb{F}_{q}$, with Lie algebra $\mathfrak{L}$, and assume the characteristic $p$ of $\mathfrak{f}$ is an odd good prime for $\mathbf{G}$.

Let $F$ be the Frobenius map on $G$ and $\mathfrak{L}$, arising from the given $\mathfrak{f}$-structure. (Here $F$ plays the role of the twisted Frobenius $F_{f}$ in Remark 2 of Section 4.2.)

Let $\mathfrak{t} \subset \mathfrak{b}$ be a Cartan subalgebra contained in a Borel subalgebra of $\mathfrak{L}$, such that $F(\mathfrak{t})=\mathfrak{t}$ and $F(\mathfrak{b})=\mathfrak{b}$. The action of $F$ on $\mathfrak{t}$ induces an automorphism $\phi \in \operatorname{Aut}(R, \Delta)$, where $(X, \Delta, \check{X}, \check{\Delta})$ is the based root datum of $\mathrm{G}$. We may extend $\phi$ to a pinned algebraic automorphism of G (denoting the extension again by $\phi$ ) and write

$$
F=\phi F_{0},
$$

where $F_{0}$ is the Frobenius map for the split $\mathfrak{f}$-structure on $\mathrm{G}$. Let $N$ and $T$ be the normalizer and centralizer of $\mathfrak{t}$ in $\mathrm{G}$, with Weyl group $W=N / T$.

Let $\theta$ be an automorphism of $\mathfrak{L}$ whose order $m$ is nonzero in $\mathfrak{f}$ such that

$$
F \circ \theta=\theta^{q} \circ F
$$

as maps $\mathfrak{L} \rightarrow \mathfrak{L}($ cf. (4.12) $)$. Then $\theta=\check{\lambda}(\zeta) \vartheta$, where $\zeta \in \mathfrak{F}^{\times}$is a root of unity of order $m$ and $\vartheta$ is a pinned automorphism of $\mathfrak{L}$ arising from an element of $\operatorname{Aut}(R, \Delta)$, which we also denote by $\vartheta$.

Set $G_{0}=\left(G^{\theta}\right)^{\circ}$ and let $\vee$ be the $\zeta$-eigenspace of $\theta$ in $\mathfrak{L}$. Condition (4.12) implies that $F\left(\mathrm{G}_{0}\right)=\mathrm{G}_{0}, F(\mathrm{~V})=\mathrm{V}$, and the representation of $\mathrm{G}_{0}$ on $\mathrm{V}$ is defined over $\mathfrak{f}$. By the Lang-Steinberg theorem for $\mathrm{G}_{0}$ there exists a Cartan subspace $\mathfrak{c}$ in $\mathrm{V}$ for which $F(\mathfrak{c})=\mathfrak{c}$. The groups

$$
N_{\mathfrak{c}}=N_{\mathrm{G}_{0}}(\mathfrak{c}), \quad S_{\mathfrak{c}}=C_{\mathrm{G}_{0}}(\mathfrak{c}), \quad W(\mathfrak{c}, \theta)=N_{\mathfrak{c}} / S_{\mathfrak{c}}
$$

are also preserved by $F$.

The relation (6.1) also implies that

$$
\phi \vartheta=\vartheta^{q} \phi \in \operatorname{Aut}(R, \Delta) .
$$


Assume that $\mathrm{G}_{0}$ has stable orbits in $\mathrm{V}$ and let $\Sigma_{m} \subset W \vartheta$ be the unique $W$-conjugacy class of $\mathbb{Z}$-regular elliptic elements of order $m$ (see Section 5 ). We consider the set of pairs

$$
M(\vartheta, \phi)=\left\{(\sigma, \tau) \in \Sigma_{m} \times W \phi: \tau \sigma \tau^{-1}=\sigma^{q}\right\},
$$

on which $W$ acts by conjugation in both factors. Given $(\sigma, \tau) \in M(\vartheta, \phi)$, we have a twisted Frobenius map

$$
F_{\tau}:=\tau \circ F_{0}
$$

on $\mathfrak{t}$, preserving the eigenspace

$$
\mathfrak{t}(\sigma, \zeta):=\{v \in \mathfrak{t}: \sigma(v)=\zeta v\} .
$$

We define a map

$$
\{\mathfrak{c}: F(\mathfrak{c})=\mathfrak{c}\} / \mathrm{G}_{0}^{F} \longrightarrow M(\vartheta, \phi) / W
$$

from the set of $\mathrm{G}_{0}^{F}$-orbits of Cartan subspaces in $\mathrm{V}$ preserved by $F$ to the set of $W$-conjugacy classes in $M(\vartheta, \phi)$, as follows.

Since $G_{0}$ has stable orbits in $V$, it follows [32, Prop. 1] that $\mathfrak{c}$ contains regular elements in $\mathfrak{L}$. The centralizer $\mathfrak{s}=\mathfrak{z}(\mathfrak{c})$ is a Cartan subalgebra of $\mathfrak{L}$ so there exists $g \in \mathrm{G}$ such that $\mathfrak{s}=\operatorname{Ad}(g) \mathfrak{t}$. Now $\mathfrak{s}$ and $\mathfrak{t}$ are both preserved by $F$, so we have $g^{-1} F(g) \in N$, projecting to an element $u \in W$ and we set $\tau=u \phi \in W \phi$. Then $\operatorname{Ad}(g): \mathfrak{t} \rightarrow \mathfrak{s}$ intertwines the action of $F$ on $\mathfrak{s}$ with the action of $F_{\tau}$ on $\mathfrak{t}$.

The automorphism

$$
\theta^{\prime}:=\operatorname{Ad}(g)^{-1} \circ \theta \circ \operatorname{Ad}(g)
$$

preserves $\mathfrak{t}$ and by restriction gives an automorphism $\sigma:=\left.\theta^{\prime}\right|_{\mathfrak{t}} \in \Sigma_{m}$. The relation (4.12) implies that $(\sigma, \tau) \in M(\vartheta, \phi)$. A different choice of $g$ such that $\mathfrak{s}=\operatorname{Ad}(g) \mathfrak{t}$ leads to a $W$-conjugate of $(\sigma, \tau)$, and replacing $\mathfrak{c}$ by a $\mathrm{G}_{0}^{F}$-conjugate of $\mathfrak{c}$ leads to the same pair $(\sigma, \tau)$. Thus, sending $\mathfrak{c} \mapsto(\sigma, \tau)$ gives the well-defined map (6.3). It has the following property.

Lemma 6.1. If, under the map [6.3), the $\mathrm{G}_{0}^{F}$-orbit of $\mathfrak{c}$ corresponds to the $W$ conjugacy class of $(\sigma, \tau)$, then there exist isomorphisms

$$
\gamma: W(\mathfrak{c}, \theta) \stackrel{\sim}{\longrightarrow} C_{W}(\sigma), \quad \delta: \mathfrak{c} \stackrel{\sim}{\longrightarrow} \mathfrak{t}(\sigma, \zeta),
$$

where $C_{W}(\sigma)$ is the centralizer of $\sigma$ in $W$, such that for all $w \in W(\mathfrak{c}, \theta)$ the map $\delta$ intertwines the action of $w F$ on $\mathfrak{c}$ with the action of $\gamma(w) F_{\tau}$ on $\mathfrak{t}(\sigma, \tau)$.

Proof. The map $\operatorname{Ad}(g)$ in the definition of (6.3) intertwines the pairs $(\mathfrak{s}, F)$ and $\left(\mathfrak{t}, F_{\tau}\right)$, along with the actions of $\theta$ on $\mathfrak{s}$ and $\sigma$ on $\mathfrak{t}$, and induces an isomorphism $C_{W(\mathfrak{s})}(\theta) \stackrel{\sim}{\longrightarrow} C_{W}(\sigma)$. Also, from [32, Cor. 21] we have $C_{W(\mathfrak{s})}(\theta)=W(\mathfrak{c}, \theta)$. Thus, $\operatorname{Ad}(g)$ induces maps $\gamma$ and $\delta$ as in the assertion of the lemma.

\section{LANGLANDS PARAMETERS FOR EPIPELAGIC REPRESENTATIONS}

We return to the setting of $p$-adic groups and assume that our connected $k$-group $G$ is absolutely simple and also simply connected. Recall that the $k$-structure on the quasi-split $k$-form of $G$ is determined by a homomorphism

$$
\varrho: \operatorname{Gal}(\bar{k} / k) \longrightarrow \operatorname{Aut}(R, \Delta)
$$

whose image $\Theta$ is generated by elements $\vartheta, \phi \in \operatorname{Aut}(R, \Delta)$ satisfying $\phi \vartheta=\vartheta^{q} \phi$, where $\vartheta$ generates the image of the inertia subgroup of $\operatorname{Gal}(\bar{k} / k)$ and has order prime to $p$ and where $\phi$ is the image of a Frobenius element $\left(a \mapsto a^{q}\right)$ in $\operatorname{Gal}(\bar{k} / k)$. 
Let $\hat{G}$ be a connected adjoint Lie group whose root datum is dual to that of $G$, and let $\hat{\mathfrak{g}}$ be the Lie algebra of $\hat{G}$. Then $\hat{G}=\operatorname{Aut}(\hat{\mathfrak{g}})^{\circ}$ is the identity component of the automorphism group of the complex simple Lie algebra $\hat{\mathfrak{g}}$. Fix a pinning $\left(\hat{T}, \hat{B},\left\{\hat{e}_{\alpha}\right\}\right)$ in $\hat{G}$, consisting of a maximal torus $\hat{T}$ contained in a Borel subgroup $\hat{B}$, along with simple root vectors $\hat{e}_{\alpha}$ for each $\check{\alpha} \in \check{\Delta}$. We identify $W=\hat{N} / \hat{T}$, where $\hat{N}$ is the normalizer of $\hat{T}$ in $\hat{G}$.

The group $\operatorname{Aut}(R, \Delta)$ acts on $\hat{G}$ via pinned automorphisms and we have $\hat{G} \rtimes$ $\operatorname{Aut}(R, \Delta)=\operatorname{Aut}(\hat{\mathfrak{g}})$. We set

$$
{ }^{L} G:=\hat{G} \rtimes \Theta \subset \operatorname{Aut}(\hat{\mathfrak{g}})
$$

and let pr: ${ }^{L} G \rightarrow \Theta$ be the projection along $\hat{G}$.

We will construct discrete Langlands parameters attached to our epipelagic representations $\pi_{x}(\lambda, \rho)$. These will be continuous homomorphisms

$$
\varphi: \mathcal{W}_{k} \longrightarrow{ }^{L} G
$$

from the Weil group of $k$, for which $\operatorname{pr} \circ \varphi=\varrho$ and which have the expected properties of the adjoint Swan conductor $b(\varphi)$ and centralizer $A_{\varphi}=C_{\hat{G}}(\varphi)$.

We first show how these properties impose strong conditions on $\varphi$. Then we give a template for the construction of parameters $\varphi$ satisfying these conditions, and we use the template in two situations: First, when $p$ is a nontorsion prime for $G$ not dividing $2 m$, we will attach a discrete parameter to each stable $\mathrm{G}_{0}(\mathfrak{f})$-orbit of stable vectors in $\check{\mathrm{V}}_{x, 1 / m}(\mathfrak{f})$. Here the image of $\varphi$ will lie in the normalizer ${ }^{L} N$ of $\hat{T}$ in ${ }^{L} G$. Then we will consider some cases where $p$ divides $2 m$ or is a torsion prime for $G$; here Theorem 4.1 does not apply but stable orbits persist. In these cases the image of $\varphi$ will lie in the normalizer in ${ }^{L} G$ of a Jordan subgroup of $\hat{G}$.

7.1. Predictions for the Langlands parameter. According to the conjectural local Langlands correspondence, each epipelagic representation $\pi_{x}(\lambda, \rho)$ should correspond to a Langlands parameter (see [14, Section 7])

$$
\varphi: \mathcal{W}_{k} \times \mathrm{SL}_{2}(\mathbb{C}) \longrightarrow{ }^{L} G
$$

whose image has finite centralizer $A_{\varphi}=C_{\hat{G}}(\varphi)$, along with an irreducible projective representation $\xi$ of $A_{\varphi}$, such that certain numerical invariants of the representation $\pi_{x}(\lambda, \rho)$ and the pair $(\varphi, \xi)$ agree. In particular, the adjoint gamma value of $\varphi$ should be related to the formal degree of $\pi_{x}(\lambda, \rho)$, as proposed in [17. As reformulated in [14], this expected property is the following.

Let $\mu_{G}$ be the absolute value of the Euler-Poincaré measure on $G(k)$ [14, 7.1]. We write $\operatorname{Deg}(\pi)=\operatorname{deg}_{\mu_{G}}(\pi)$ for the formal degree with respect to $\mu_{G}$ of a discrete series representation $\pi$. Then the pair $(\varphi, \xi)$ attached to $\pi_{x}(\lambda, \rho)$ should satisfy

$$
\operatorname{Deg}\left(\pi_{x}(\lambda, \rho)\right) \stackrel{?}{=} \frac{\operatorname{dim} \xi}{\left|A_{\varphi}\right|} \cdot \frac{\omega\left(\varphi_{0}\right)}{\gamma\left(\varphi_{0}\right)} \cdot \frac{\gamma(\varphi)}{\omega(\varphi)},
$$

where $\gamma(\varphi)=\gamma(\varphi, \hat{\mathfrak{g}}, 0)$ is the gamma value at $s=0$ of the adjoint representation

$$
\mathcal{W}_{k} \times \mathrm{SL}_{2}(\mathbb{C}) \stackrel{\varphi}{\longrightarrow}{ }^{L} G \stackrel{\operatorname{Ad}}{\longrightarrow} \operatorname{Aut}(\hat{\mathfrak{g}}),
$$

$\omega(\varphi)$ is the adjoint root number, and $\varphi_{0}: \mathcal{W}_{k} \times \mathrm{SL}_{2}(\mathbb{C}) \rightarrow{ }^{L} G$ is the principal parameter (which corresponds to the Steinberg representation of $G(k)$ ). The righthand side of (7.1) is of the form $\Gamma_{\varphi}(q)$ where $\Gamma_{\varphi}(x) \in \mathbb{Q}(x)$ is a rational function [14, Prop. 4.1]. 
We now analyze the conditions that equality in (7.1) would impose on $\gamma(\varphi) / \omega(\varphi)$. These will lead to strong conditions on $\varphi$ itself.

Let $y \in \mathcal{A}(S, k)$ be a point such that $G(k)_{y, 0}$ is an Iwahori subgroup of $G(k)$ contained in $G(k)_{x, 0}$. Then the compact open subgroup $J_{y}=G(k)_{y, 0+}$ contains $J_{x}$. From [14, (55)], the volume of $J_{y}$ with respect to the measure $\mu_{G}$ can be expressed as

$$
\operatorname{vol}\left(J_{y}\right)=q^{-\left(\operatorname{dim} G+\ell_{\vartheta}\right) / 2} \cdot \frac{\gamma\left(\varphi_{0}\right)}{\omega\left(\varphi_{0}\right)},
$$

where we recall that $\ell_{\vartheta}$ is the rank of $G$ over $K$. It follows that

$$
\operatorname{vol}\left(J_{x}\right)=\operatorname{vol}\left(J_{y}\right) \cdot\left[J_{y}: J_{x}\right]^{-1}=q^{-\left(\operatorname{dim} G+\operatorname{dim} G_{x}\right) / 2} \cdot \frac{\gamma\left(\varphi_{0}\right)}{\omega\left(\varphi_{0}\right)} .
$$

From Proposition 2.4, the formal degree of $\pi_{x}(\lambda, \rho)$ with respect to $\mu_{G}$ is (actually) given by

$$
\operatorname{Deg}\left(\pi_{x}(\lambda, \rho)\right)=\frac{\operatorname{dim} \chi_{\lambda, \rho}}{\left|A_{x, \lambda}\right|} \cdot q^{\left(\operatorname{dim} G+\operatorname{dim} \mathrm{G}_{x}\right) / 2} \cdot \frac{\omega\left(\varphi_{0}\right)}{\gamma\left(\varphi_{0}\right)} .
$$

Equality of the right sides of (7.2) and (7.1) forces the $L$-function of $\varphi$ to be trivial, so that $\varphi$ is totally ramified (that is, $\hat{\mathfrak{g}}^{\varphi(\mathcal{I})}=0$, where $\mathcal{I} \subset \mathcal{W}_{k}$ is the inertia subgroup) and then $\varphi$ is trivial on $\mathrm{SL}_{2}(\mathbb{C})$ and we have [14, 3.2]

$$
\frac{\gamma(\varphi)}{\omega(\varphi)}=q^{\alpha(\varphi) / 2}
$$

where $\alpha(\varphi)$ is the Artin conductor of the representation Ad $\circ \varphi$ on $\hat{\mathfrak{g}}$. Therefore equality in (7.1) forces $\alpha(\varphi)=\operatorname{dim} G+\operatorname{dim} \mathrm{G}_{x}$. But, again since $\varphi$ is totally ramified, we have $\alpha(\varphi)=\operatorname{dim} \hat{\mathfrak{g}}+b(\varphi)=\operatorname{dim} G+b(\varphi)$, where $b(\varphi)$ is the Swan conductor of Ad $\circ \varphi$. Thus, we find that equality in (7.1) forces

$$
b(\varphi)=\operatorname{dim} G_{x}
$$

and

$$
\frac{\operatorname{dim} \xi}{\left|A_{\varphi}\right|}=\frac{\operatorname{dim} \chi_{\lambda, \rho}}{\left|A_{x, \lambda}\right|}
$$

Conversely, it is clear that (7.3) and (7.4) imply that equality holds in (7.1).

If $p$ does not divide $|W|$, we can sharpen the predicted equality (7.3) and show that it is an extremal condition on $\varphi$, as follows. Let $\varphi(\mathcal{I})=D_{0} \geq D_{1} \geq D_{2} \geq \cdots$ be the lower ramification filtration of the inertial image of $\varphi$. Since $p \nmid|W|$, it follows from [4] that $D_{1}$ is contained in a maximal torus of $\hat{G}$, which we may assume is $\hat{T}$, and that $D_{0}$ is contained in the normalizer ${ }^{L} N$ of $\hat{T}$ in ${ }^{L} G$. We therefore have $D_{0}=\left\langle\hat{n}_{0}\right\rangle \ltimes D_{1}$, where $\hat{n}_{0} \in{ }^{L} N$ is an element whose order, say $m_{0}$, is prime to $p$. Let $\sigma_{0}$ be the projection of $\hat{n}_{0}$ to $W \vartheta$. Since $\hat{\mathfrak{g}}^{D_{0}}=0$, it follows that $\hat{\mathfrak{g}}^{D_{1}}=\hat{\mathfrak{t}}$ and that $\hat{\mathfrak{t}}^{\hat{\sigma}_{0}}=0$ [14, Prop. 5.4]. Then from the definition of the Swan conductor we have

$$
b(\varphi) \geq \frac{\operatorname{dim}(\hat{\mathfrak{g}} / \hat{\mathfrak{t}})}{m_{0}}=\frac{|R|}{m_{0}},
$$

with equality if and only if $D_{2}=1$.

On the other hand, via the Kac classification (see Section 3.3), the automorphism $\operatorname{Ad}\left(\hat{n}_{0}\right) \in \operatorname{Aut}(\hat{\mathfrak{g}})$ corresponds to a point $y \in \mathcal{A}(T, K)$ such that $\left(\hat{G}^{\mathrm{Ad}\left(\hat{n}_{0}\right.}\right)^{\circ}$ and $\mathrm{G}_{y}$ 
are reductive groups (over $\mathbb{C}$ and $\mathfrak{f}$, respectively) with the same root data. Since $\hat{\mathfrak{t}}^{\sigma_{0}}=0$, it follows that

$$
\operatorname{dim} \mathrm{G}_{y}=\operatorname{dim} \hat{\mathfrak{g}}^{\operatorname{Ad}\left(\hat{n}_{0}\right)} \geq\left|R / \sigma_{0}\right| \geq \frac{|R|}{m_{0}},
$$

with equality if and only if $\left\langle\sigma_{0}\right\rangle$ acts freely on $R$.

If $D_{2}=1$ and $\sigma_{0}$ is a $\mathbb{Z}$-regular automorphism of $R$, the lower bounds in these inequalities will be attained and then (17.3) will be satisfied exactly when $y \in W_{\text {aff }}(R, \vartheta) \cdot x$. This last condition means that $m_{0}=m$ is the order of $x$ and the tame inertial generator $\hat{n}_{0}$ of the discrete parameter $\varphi$ induces a $\mathbb{Z}$-regular elliptic automorphism $\sigma_{0}$ of $R$ which is $W$-conjugate to the automorphism $\sigma$ corresponding to $x$ as in Section 5 .

7.2. A template for epipelagic parameters. Recall that $e$ is the ramification degree of the minimal tame extension splitting $G$. Let $m, f$ be positive integers such that $e$ divides $m$ and $m$ divides $q^{f}-1$.

We make some slight changes in notation. Let $K$ and $K_{m}$ now denote the unramified extensions of $k$ of degrees $f$ and $m f$, respectively, and let $\mathfrak{F}, \mathfrak{F}_{m}$ be the residue fields of $K, K_{m}$. Let $M / K$ be the abelian extension with norm group $N(M / K)=K^{\times m}$. We choose a root $\pi=\sqrt[m]{\varpi}$, where $\varpi$ is a prime element in $A_{k}$. Then $M=K_{m}(\pi)$, and $M / k$ is a tamely ramified Galois extension of degree $[M: k]=f m^{2}$. The Galois group $\Gamma=\operatorname{Gal}(M / k)$ has the presentation

$$
\Gamma=\left\langle s, t: s^{m}=t^{m f}=1, t s t^{-1}=s^{q}\right\rangle,
$$

where

$$
s(\pi)=\zeta \pi, \quad t(\pi)=\pi,
$$

and $s$ (resp. $t$ ) are the identity (resp. Frobenius) automorphisms on $K_{m}$. We identify $\mathfrak{F}_{m}$ with the residue field of $M$ and regard $\mathfrak{F}_{m}^{\times}$as a subgroup of $M^{\times}$. Let $U_{M}^{n}=1+\pi^{n} A_{M}$ be the higher unit groups of $M$. The choice of $\pi$ gives an isomorphism (denoted by the same letter)

$$
U_{M}^{1} / U_{M}^{2} \stackrel{\pi}{\longrightarrow} \mathfrak{F}_{m}^{+}, \quad 1+\pi a \mapsto a \bmod \pi A_{M} .
$$

With the natural action of $\Gamma$ on $U_{M}^{1} / U_{M}^{2}$ and letting $\Gamma$ act on $\mathfrak{F}_{m}$ via $s \cdot a=\zeta a$ and $t \cdot a=a^{q}$ for $a \in \mathfrak{F}_{m}$, the map $\pi$ becomes an isomorphism of $\mathbb{F}_{p}[\Gamma]$-modules.

Let $L / M$ be the abelian extension with norm group

$$
N(L / M)=\langle\pi\rangle \times \mathfrak{F}_{m}^{\times} \times U_{M}^{2},
$$

so that $\operatorname{Gal}(L / M) \simeq U_{M}^{1} / U_{M}^{2}$. Then $L / k$ is Galois, since $N(L / M)$ is preserved by $\Gamma$. Also, since $\operatorname{Gal}\left(M / K_{m}\right)=\langle s\rangle$ has trivial invariants in $U_{M}^{1} / U_{M}^{2}$, it follows [14. Lemma 6.1] that there is a splitting

$$
\operatorname{Gal}(L / k) \simeq\left(U_{M}^{1} / U_{M}^{2}\right) \rtimes \Gamma \simeq \mathfrak{F}_{m}^{+} \rtimes \Gamma,
$$

where the first isomorphism is canonical and the second depends on the choice of $\pi$, as in (7.6).

Suppose $J \subset \hat{G}$ is a finite elementary abelian $p$-subgroup of $\hat{G}$, whose normalizer ${ }^{L} N(J)$ in ${ }^{L} G$ contains two elements $\hat{n}_{s} \in \hat{G} \vartheta$ of order $m$ and $\hat{n}_{t} \in \hat{G} \phi$ satisfying $\hat{n}_{t}^{m f}=1$, with the relation $\hat{n}_{t} \hat{n}_{s} \hat{n}_{t}^{-1}=\hat{n}_{s}^{q}$.

Then we have a homomorphism

$$
\eta: \Gamma \rightarrow{ }^{L} N(J), \quad s \mapsto \hat{n}_{s}, \quad t \mapsto \hat{n}_{t},
$$


and any $\Gamma$-equivariant homomorphism $\psi: \mathfrak{F}_{m}^{+} \rightarrow J$ extends to a homomorphism

$$
\psi \rtimes \eta: \operatorname{Gal}(L / k) \simeq \mathfrak{F}_{m}^{+} \rtimes \Gamma \longrightarrow{ }^{L} N(J), \quad(a, \gamma) \mapsto \psi(a) \cdot \eta(\gamma),
$$

for $a \in \mathfrak{F}_{m}$ and $\gamma \in \Gamma$. Replacing $J$ by $\operatorname{im} \psi$ if necessary, we may assume $\psi$ is surjective. Then the Galois group $D=\operatorname{im}(\psi \rtimes \eta)$ has lower ramification filtration

$$
D=\left\langle J, \hat{n}_{s}, \hat{n}_{t}\right\rangle, \quad D_{0}=\left\langle J, \hat{n}_{s}\right\rangle, \quad D_{1}=J, \quad D_{2}=1,
$$

and the Swan conductor of the adjoint representation $\operatorname{Gal}(L / k) \stackrel{\psi \rtimes \eta}{\longrightarrow} N(J) \stackrel{\mathrm{Ad}}{\longrightarrow}$ $\operatorname{Aut}(\hat{\mathfrak{g}})$ is given by

$$
b(\psi \rtimes \eta)=\frac{\operatorname{dim}\left(\hat{\mathfrak{g}} / \hat{\mathfrak{g}}^{J}\right)}{m},
$$

where $\hat{\mathfrak{g}}^{J}$ is the fixed-point subalgebra of $J$ in $\hat{\mathfrak{g}}$. To have $\hat{\mathfrak{g}}^{D_{0}}=0$ (total ramification), $\hat{\mathfrak{g}}^{J}$ must be the Lie algebra of a torus (possibly trivial) in $\hat{G}$.

If $p$ is not a torsion prime for $\hat{G}$, then $J$ is contained in a maximal torus of $\hat{G}$, by [35, 2.28], so

in this case.

$$
b(\psi \rtimes \eta)=\frac{\operatorname{dim}(\hat{\mathfrak{g}} / \hat{\mathfrak{t}})}{m}=\frac{|R|}{m},
$$

7.3. Parameters arising from Cartan subspaces. Let $x \in \mathcal{A}(S, k)$ be a rational point of order $m$ such that $\check{\mathrm{V}}_{x}=\check{\mathrm{V}}_{x, 1 / m}$ contains stable functionals for the action of $\mathrm{G}_{x}$. In this section we will attach a discrete Langlands parameter to each $\mathrm{G}_{x}(\mathfrak{f})$ orbit of stable functionals in $\check{\mathrm{V}}_{x}(\mathfrak{f})$, assuming that the characteristic $p$ of the residue field $\mathfrak{f}$ is not a torsion prime for $\hat{G}$ and that $p \nmid 2 m$.

Let $\mathfrak{c} \subset \check{\mathfrak{V}}_{x}$ be a Cartan subspace for which $F(\mathfrak{c})=\mathfrak{c}$. Then $\mathfrak{c}$ is defined over $\mathfrak{f}$ and we have $\mathfrak{c}(\mathfrak{f})=\mathfrak{c}^{F}$. The point $x$ and choice of root of unity $\zeta \in \mathfrak{F}^{\times}$determine an automorphism $\theta$ (see (4.4) $)$ of the Lie algebra $\mathfrak{L}=\operatorname{Lie}(\mathrm{G})$ and from Theorem 4.1. we have an isomorphism

$$
f_{1}: \check{\mathrm{V}}_{x} \stackrel{\sim}{\longrightarrow} \mathfrak{L}(\theta, \zeta)
$$

sending $\mathfrak{c}$ to the eigenspace $\mathfrak{s}(\theta, \zeta)$, where $\mathfrak{s}$ is the unique Cartan subalgebra of $\mathfrak{L}$ centralizing $f_{1}(\mathfrak{c})$. The $\mathrm{G}$-invariant pairing on $\mathfrak{L}$ restricts to a pairing

$$
\mathfrak{s}\left(\theta, \zeta^{-1}\right) \otimes \mathfrak{c} \simeq \mathfrak{s}\left(\theta, \zeta^{-1}\right) \otimes \mathfrak{s}(\theta, \zeta) \longrightarrow \mathfrak{F} .
$$

We identify the Cartan subalgebra $\mathfrak{t}$ of Section 6.1 with $\mathfrak{F} \otimes \check{X}$; its dual space is $\hat{\mathfrak{t}}:=\mathfrak{F} \otimes X$. Recall that the map (6.3) determines a $W$-congugacy class of pairs $(\sigma, \tau) \in M(\vartheta, \phi)$, arising from an element $g \in \mathrm{G}$ such that $\operatorname{Ad}(g) \mathfrak{t}=\mathfrak{s}$, and $\operatorname{Ad}(g)$ interwines $\sigma$ and $F_{\tau}$ on $\mathfrak{t}$ with $\theta$ and $F$ on $\mathfrak{s}$. In particular, $\operatorname{Ad}(g)$ gives an isomorphism $\mathfrak{t}\left(\sigma, \zeta^{-1}\right) \rightarrow \mathfrak{s}\left(\theta, \zeta^{-1}\right)$. By duality, we get an isomorphism

$$
\mathfrak{c} \stackrel{\sim}{\longrightarrow} \hat{\mathfrak{t}}(\sigma, \zeta),
$$

which intertwines $F$ on $\mathfrak{c}$ and $F_{\tau}$ on $\hat{\mathfrak{t}}(\sigma, \zeta)$. Thus, we get an isomorphism on rational points

$$
\mathfrak{c}(\mathfrak{f})=\mathfrak{c}^{F} \stackrel{\sim}{\longrightarrow} \hat{\mathfrak{t}}(\sigma, \zeta)^{F_{\tau}} .
$$

Recall that $\sigma$ is a $\mathbb{Z}$-regular elliptic element in $\operatorname{Aut}(R, \Delta)$. Since $\hat{G}$ is adjoint, all lifts of $\sigma$ in ${ }^{L} N$ are $\hat{T}$-conjugate and have order $m$ [32, Prop. 8]. We fix one such lift $\hat{n}_{\sigma} \in{ }^{L} N$. For any lift $\hat{n}_{\tau}^{\prime} \in{ }^{L} N$ of $\tau$, we will have $\hat{n}_{\tau}^{\prime} \cdot \hat{n}_{\sigma} \cdot\left(\hat{n}_{\tau}^{\prime}\right)^{-1} \cdot y=\hat{n}_{\sigma}^{q}$, for 
some $y \in \hat{T}$. By the ellipticity of $\sigma$, there exists $z \in \hat{T}$ such that $z^{\sigma} \cdot z^{-1}=y^{\tau}$. We choose the new lift $\hat{n}_{\tau}:=\hat{n}_{\tau}^{\prime} \cdot z$, so that $\hat{n}_{\tau} \cdot \hat{n}_{\sigma} \cdot \hat{n}_{\tau}^{-1}=\hat{n}_{\sigma}^{q}$.

Let $f$ be the order of $\tau$ in $W$. Then $\sigma=\tau^{f} \sigma \tau^{-f}=\sigma^{q^{f}}$, so $m \mid q^{f}-1$ and $\hat{\tau}^{f} \in \hat{T}^{\sigma}$. The latter is a finite abelian group of exponent dividing $m$ [31, 2.1], so $\hat{\tau}^{f m}=1$. As in Section 7.2 we have a homomorphism $\eta: \Gamma \longrightarrow{ }^{L} N$, sending $s \mapsto \hat{n}_{\sigma}$ and $t \mapsto \hat{n}_{\tau}$. This gives an action of $\Gamma$ on $\hat{T}$, whereby $s$ acts via $\sigma$ and $t$ acts via $\tau$.

Let $\hat{T}[p]:=\left\{t \in \hat{T}: t^{p}=1\right\}$ be the $p$-torsion subgroup of $\hat{T}$. Since $X=$ $\operatorname{Hom}\left(\mathbb{C}^{\times}, \hat{T}\right)$, we have an isomorphism

$$
\mathbb{F}_{p} \otimes X \stackrel{\sim}{\longrightarrow} \hat{T}[p], \quad a \otimes \lambda \mapsto \lambda\left(z^{a}\right),
$$

where $z=\exp (2 \pi \sqrt{-1} / p)$.

Let $h(x) \in \mathbb{F}_{p}[x]$ be the minimal polynomial of $\zeta$ over $\mathbb{F}_{p}$. The group $J$ of Section 7.2 will be the subgroup

$$
\hat{T}[p, \zeta]:=\{y \in \hat{T}[p]: h(\sigma) y=1\},
$$

which we will henceforth identify with the kernel of $h(\sigma)$ in $\mathbb{F}_{p} \otimes X$, via (7.11). This subspace of $\hat{T}[p]$ is preserved by $\sigma$ and $\tau$. Thus, $\hat{T}[p, \zeta]$ becomes an $\mathbb{F}_{p}[\Gamma]$ module, on which $t^{f}$ acts trivially.

Lemma 7.1. Let $\operatorname{tr}: \mathfrak{F}_{m} \rightarrow \mathbb{F}_{p}$ be the trace map. There is a canonical isomorphism

$$
\hat{\mathfrak{t}}(\sigma, \zeta)^{F_{\tau}} \simeq \operatorname{Hom}_{\Gamma}\left(\mathfrak{F}_{m}, \hat{T}[p, \zeta]\right), \quad \lambda \mapsto \psi_{\lambda}^{\prime},
$$

such that $\left\langle\psi_{\lambda}^{\prime}(b), \check{\omega}\right\rangle=\operatorname{tr}(b\langle\lambda, \check{\omega}\rangle)$, for all $b \in \mathfrak{F}_{m}$ and $\check{\omega} \in \check{X}$.

Proof. Nondegeneracy of the form $(a, b)=\operatorname{tr}(a b)$ implies that the map $\lambda \mapsto \psi_{\lambda}^{\prime}$ gives an isomorphism

$$
\mathfrak{F}_{m} \otimes X \stackrel{\sim}{\longrightarrow} \operatorname{Hom}_{\mathbb{F}_{p}}\left(\mathfrak{F}_{m}, \hat{T}[p]\right) .
$$

Any $F_{\tau}$-fixed element of $\hat{\mathfrak{t}}$ lies in $\mathfrak{F}_{m} \otimes X$. One checks that for all $b \in \mathfrak{F}_{m}$ we have $F_{\tau}(\lambda)=\lambda$ if and only if $\psi_{\lambda}^{\prime}\left(b^{q}\right)=\tau\left(\psi_{\lambda}^{\prime}(b)\right)$ and that $\sigma(\lambda)=\zeta \lambda$ if and only if $\psi_{\lambda}^{\prime}(\zeta b)=\sigma\left(\psi_{\lambda}^{\prime}(b)\right)$. The lemma follows.

Combining Lemma 7.1 with (7.10), we obtain an isomorphism

$$
\mathfrak{c}(\mathfrak{f}) \stackrel{\sim}{\longrightarrow} \operatorname{Hom}_{\Gamma}\left(\mathfrak{F}_{m}, \hat{T}[p, \zeta]\right),
$$

which we denote by $\lambda \mapsto \psi_{\lambda}$, for $\lambda \in \mathfrak{c}(\mathfrak{f})$.

Lemma 7.2. If $\lambda \in \mathfrak{c}(\mathfrak{f})$ is stable, then $\hat{T}$ is the full centralizer in $\hat{G}$ of the image $\psi_{\lambda}\left(\mathfrak{F}_{m}\right)$.

Proof. A stable vector $\lambda \in \mathfrak{c}$ is a regular semisimple element in $\mathfrak{L}=\operatorname{Lie}(\mathrm{G})$ 32, Lemma 13]. As above, let $\mathfrak{s}$ be the Cartan subalgebra centralizing $\lambda$ and let $g \in \mathrm{G}$ be such that $\operatorname{Ad}(g) \mathfrak{t}=\mathfrak{s}$. Then $\operatorname{Ad}\left(g^{-1}\right) \lambda \in \mathfrak{t}$ has trivial stabilizer in $W(\mathfrak{t})$, the Weyl group of $\mathfrak{t}$ in $\mathrm{G}$. The isomorphism $\mathfrak{t} \simeq \hat{\mathfrak{t}}$ induced by the $\mathrm{G}$-invariant pairing on $\mathfrak{L}$ is $W$-equivariant, so the isomorphism (7.9) sends $\lambda$ to an element $\lambda^{\prime} \in \hat{\mathfrak{t}}(\sigma, \zeta)$ with trivial stabilizer in $W(\mathfrak{t})$. This means that $\left\langle\lambda^{\prime}, \check{\alpha}\right\rangle \neq 0$ for all co-roots $\check{\alpha} \in \check{R}$. Hence $\left\langle\psi_{\lambda}(b), \check{\alpha}\right\rangle=\operatorname{tr}\left(b\left\langle\lambda^{\prime}, \check{\alpha}\right\rangle\right) \neq 0$ for some $b \in \mathfrak{F}_{m}$. Since $\check{R}$ is the set of roots of $\hat{T}$ in $\hat{G}$ and we have assumed $p$ is not a torsion prime for $\hat{G}$, it follows from [35, 2.25] that $\hat{T}$ is the full centralizer of $\psi_{\lambda}(\mathfrak{F})$, as claimed. 
Now take $\lambda \in \mathfrak{c}(\mathfrak{f})$ to be a stable vector, with corresponding map

$$
\psi_{\lambda} \in \operatorname{Hom}_{\Gamma}\left(\mathfrak{F}_{m}, \hat{T}[p, \zeta]\right),
$$

as in (7.12). As in (7.8) we define

$$
\varphi_{\lambda}:=\psi_{\lambda} \rtimes \eta: \operatorname{Gal}(L / k) \longrightarrow{ }^{L} N \subset{ }^{L} G,
$$

and we set

$$
A_{\varphi_{\lambda}}=C_{\hat{G}}\left(\varphi_{\lambda}\right) .
$$

From Lemma 7.2, we have $C_{\hat{G}}\left(\operatorname{im} \psi_{\lambda}\right)=\hat{T}$, so

$$
A_{\varphi_{\lambda}}=\hat{T}^{\sigma, \tau}=\hat{T}^{\Gamma} .
$$

Since $\sigma$ is elliptic, the group $\hat{T}^{\sigma}$ is finite, so $\varphi_{\lambda}$ is a discrete Langlands parameter whose image $D=\operatorname{im} \varphi_{\lambda} \subset{ }^{L} N$ has lower filtration groups

$$
D_{0}=\left\langle\hat{n}_{\sigma}\right\rangle \rtimes \operatorname{im} \psi_{\lambda}, \quad D_{1}=\operatorname{im} \psi_{\lambda}, \quad D_{2}=1 .
$$

The Swan conductor of the adjoint representation

$$
\operatorname{Ad} \circ \varphi_{\lambda}: \operatorname{Gal}(L / k) \longrightarrow{ }^{L} G=\operatorname{Aut}(\hat{\mathfrak{g}})
$$

is therefore given by

$$
b\left(\varphi_{\lambda}\right)=\frac{\operatorname{dim} \hat{\mathfrak{g}}-\operatorname{dim} \hat{\mathfrak{t}}}{\left[D_{0}: D_{1}\right]}=\frac{|R|}{m}=\operatorname{dim} \hat{\mathfrak{g}}^{\hat{n}_{\sigma}}=\operatorname{dim} \mathrm{G}_{0} .
$$

Since $\mathrm{G}_{0}$ is isomorphic to the reductive quotient $\mathrm{G}_{x}$ of the parahoric subgroup $G(K)_{x, 0}$, the expected equality (7.3) holds for $\varphi_{\lambda}$.

Finally, since $G$ is simply connected, so is the group $\mathrm{G}$. It follows that $\mathrm{G}_{0}=\mathrm{G}^{\theta}$, so the stabilizer $\left\{g \in \mathrm{G}_{0}: g \cdot \lambda=\lambda\right\}=\mathrm{S}^{\theta}$, where $\mathrm{S}=C_{\mathrm{G}}(\mathfrak{c})$ is the maximal torus of $\mathrm{G}$ with Lie algebra $\mathfrak{s}$. The map $\operatorname{Ad}(g): \mathfrak{t} \rightarrow \mathfrak{s}$ (see (6.1)) gives an isomorphism $\mathrm{T}^{\sigma} \rightarrow \mathrm{S}^{\theta}$ intertwining $F$ on $\mathrm{S}^{\theta}$ with $F_{\tau}$ on $\mathrm{T}^{\sigma}$.

It follows that the group $A_{x, \lambda}$ (see (2.5) ) is given by

$$
A_{x, \lambda}=\left(\mathrm{S}^{\theta}\right)^{F} \simeq\left(\mathrm{T}^{\sigma}\right)^{F_{\tau}} .
$$

This group has the same cardinality as the invariants of $F_{\tau}^{*}$ in the coinvariants $X_{\sigma}$, where $X=X^{*}(\mathrm{~T})$, and $F_{\tau}^{*}$, the adjoint of $F_{\tau}$, acts on $X$ via $q \tau^{-1}$. It follows that

$$
\left|A_{x, \lambda}\right|=\left|X_{\sigma}^{\tau=q}\right| \text {. }
$$

On the other hand, we have isomorphisms

$$
\hat{T}^{\sigma} \stackrel{\exp }{\longleftarrow}(1-\sigma)^{-1} X / X \stackrel{1-\sigma}{\longrightarrow} X_{\sigma} .
$$

The relation $\tau \sigma \tau^{-1}=\sigma^{q}$ implies

$$
\tau^{-1} \sigma_{q} \circ(1-\sigma)=(1-\sigma) \circ \tau^{-1},
$$

where $\sigma_{q}:=1+\sigma+\cdots+\sigma^{q-1}$ acts via $q$ on $X_{\sigma}$. Thus, $1-\sigma$ intertwines $q \tau^{-1}$ on $X_{\sigma}$ with $\tau^{-1}$ on $\hat{T}^{\sigma}$, and we have

$$
\left|X_{\sigma}^{\tau=q}\right|=\left|\hat{T}^{\sigma, \tau}\right|=\left|A_{\varphi_{\lambda}}\right| .
$$

Combining (7.13) and (7.14), we obtain the equality

$$
\left|A_{x, \lambda}\right|=\left|A_{\varphi_{\lambda}}\right|
$$

between the cardinalities of the isotropy group in $\mathrm{G}_{0}$ of the stable functional $\lambda \in \mathfrak{c}(\mathfrak{f})$ and the centralizer in $\hat{G}$ of the corresponding discrete parameter $\varphi_{\lambda}$. As both $A_{x, \lambda}$ 
and $A_{\varphi_{\lambda}}$ are abelian, we have shown that the expected property (7.4) holds for the parameter $\varphi_{\lambda}$.

7.4. Simple supercuspidal parameters for $\mathrm{SU}_{p}\left(\mathbb{Q}_{p}\right)$. Let $p=2 n+1$ be an odd prime. In this section we construct parameters for the simple supercuspidal representations (Section 2.6) of the special unitary group $G=\operatorname{SU}_{p}\left(E / \mathbb{Q}_{p}\right)$ splitting over a ramified quadratic extension $E / \mathbb{Q}_{p}$.

The point $x \in \mathcal{A}(S, k)$ is the barycenter of an alcove, with all Kac coordinates $s_{i}=1$, and has order $h_{\vartheta}=2 p$. The group $\mathrm{G}_{x}$ is a maximal torus in the orthogonal group $\mathrm{SO}_{p}\left(\overline{\mathbb{F}}_{p}\right)$ and $\check{\mathrm{V}}_{x, 1 / 2 p}$ is a direct sum of lines whose characters are the gradients of the simple affine roots $-2 x_{1}, x_{1}-x_{2}, \ldots, x_{n-1}-x_{n}, x_{n}$. The stable points are those whose component in each line is nonzero and they have trivial isotropy groups in $\mathrm{G}_{x}$. It follows that there are $p-1$ simple supercuspidal representations of $G(k)$.

Let $M / \mathbb{Q}_{p}$ be the tame extension constructed in Section 7.2 , for $m=2(p+1)$ and $f=2$. Here $m$ is no longer the order of the point $x$. The quadratic character $\chi: \operatorname{Gal}\left(\overline{\mathbb{Q}}_{p} / \mathbb{Q}_{p}\right) \rightarrow\{ \pm 1\}$ corresponding to $E$ factors through the Galois group $\Gamma=\operatorname{Gal}\left(M / \mathbb{Q}_{p}\right)=\langle s, t\rangle$. Explicitly, we have $\chi(s)=-1$ (since $E / \mathbb{Q}_{p}$ is ramified) and

$$
\chi(t)=\left\{\begin{array}{lll}
+1 & \text { if } & E=\mathbb{Q}_{p}(\sqrt{p}), \\
-1 & \text { if } & E=\mathbb{Q}_{p}(\sqrt{u p}),
\end{array}\right.
$$

where $u \in \mathbb{Z}_{p}^{\times}$is a nonsquare modulo $p$.

In the dual group $\hat{G}=\operatorname{PGL}_{p}(\mathbb{C})$ there is a Jordan subgroup $J \subset{ }^{L} G$ of type $(p, p)$, generated by (the images of) matrices $A, B$ given in terms of the standard basis $\left\{e_{j}\right\}$ by

$$
A e_{j}=z^{j} e_{j}, \quad B e_{j}=e_{j+1},
$$

where $z=\exp (2 \pi \sqrt{-1} / p)$ and subscripts are read modulo $p$. The finite group $J$ is its own centralizer in $\hat{G}$ and in particular is not contained in a maximal torus of $\hat{G}$ (see [3, 6.4] or [22, Chap. 3]).

The $L$-group of $G$ is

$$
{ }^{L} G=\operatorname{PGL}_{p}(\mathbb{C}) \rtimes\langle\vartheta\rangle=\operatorname{Aut}\left(\mathfrak{s l}_{p}(\mathbb{C})\right),
$$

where $\vartheta$ is an outer pinned involution. The action on $J$ by its normalizer ${ }^{L} N(J)$ in ${ }^{L} G$ induces an isomorphism

$$
{ }^{L} N(J) / J \simeq \mathrm{SL}^{ \pm}(J)=\{g \in \mathrm{GL}(J): \operatorname{det}(g)= \pm 1\},
$$

sending the normalizer $N_{\hat{G}}(J)$ onto $\operatorname{SL}(J) \simeq \mathrm{SL}_{2}\left(\mathbb{F}_{p}\right)$.

Fix an element $\zeta \in \mathbb{F}_{p^{2}}^{\times}$of order $m=2(p+1)$. We regard $\mathbb{F}_{p^{2}}$ as a vector space over $\mathbb{F}_{p}$ and $\zeta$ as an element of $\mathrm{GL}\left(\mathbb{F}_{p^{2}}\right)$ via multiplication. Let $j: \mathbb{F}_{p^{2}} \rightarrow J$ be an isomorphism of $\mathbb{F}_{p}$-vector spaces. Up to conjugacy by $\mathrm{SL}_{2}(p)$ there are $p-1$ choices for $j$, corresponding to the value of the determinant of the change of basis matrix. Each choice of $j$ induces an isomorphism

$$
j_{*}: \mathrm{GL}\left(\mathbb{F}_{p^{2}}\right) \longrightarrow \mathrm{GL}(J)
$$

giving rise to two elements of $\operatorname{GL}(J)$ :

$$
\sigma_{j}=j_{*}(\zeta), \quad \phi_{j}=j_{*}\left(\operatorname{Frob}_{p}\right)
$$


where $\operatorname{Frob}_{p}$ is the $p^{\text {th }}$-power map on $\mathbb{F}_{p^{2}}$. One checks that $\operatorname{det}\left(\sigma_{j}\right)=\operatorname{det}\left(\phi_{j}\right)=$ $-13$

We next define

$$
\tau_{j}=\left\{\begin{array}{lll}
\sigma_{j} \phi_{j} & \text { if } & E=\mathbb{Q}_{p}(\sqrt{p}), \\
\phi_{j} & \text { if } & E=\mathbb{Q}_{p}(\sqrt{u p}) .
\end{array}\right.
$$

Then $\tau_{j} \sigma_{j} \tau_{j}^{-1}=\sigma_{j}^{p}$, and $\tau_{j}^{4}=1$, so we have a homomorphism

$$
\eta_{j}: \Gamma \longrightarrow{ }^{L} N(J), \quad s \mapsto \sigma_{j}, \quad t \mapsto \tau_{j},
$$

such that $\chi(t)=\operatorname{det}\left(\tau_{j}\right)$, as required to get a parameter for $\operatorname{SU}_{p}\left(E / \mathbb{Q}_{p}\right)$. In this way, $J$ becomes a $\Gamma$-module.

As $\zeta^{p+1}=-1$, it follows from Hilbert's Theorem 90 that $\zeta=v^{1-p}$ for some $v \in \mathbb{F}_{p^{4}}^{\times}$. The residue field $\mathfrak{F}_{m}$ of $M$ has cardinality $p^{2 m}=p^{4(p+1)}$ and therefore contains $v$. Let $\operatorname{tr}: \mathfrak{F}_{m} \rightarrow \mathbb{F}_{p^{2}}$ be the trace map and define

$$
\psi: U_{M}^{1} / U_{M}^{2} \longrightarrow \mathbb{F}_{p}^{2} \quad \text { by } \quad \psi(1+a \pi)= \begin{cases}\operatorname{tr}(v \bar{a}) & \text { if } \quad E=\mathbb{Q}_{p}(\sqrt{p}), \\ \operatorname{tr}(\bar{a}) & \text { if } \quad E=\mathbb{Q}_{p}(\sqrt{u p}) .\end{cases}
$$

One checks that the map $\psi_{j}:=j \circ \psi$ is a $\Gamma$-equivariant surjection

$$
\psi_{j}: U_{M}^{1} / U_{M}^{2} \longrightarrow J
$$

so as in Section 7.2 we have a parameter

$$
\varphi_{j}=\psi_{j} \rtimes \eta_{j}:\left(U_{M}^{1} / U_{M}^{2}\right) \rtimes \Gamma=\operatorname{Gal}\left(M / \mathbb{Q}_{p}\right) \longrightarrow{ }^{L} G .
$$

Since $J=C_{\hat{G}}(J)$, it follows that $\varphi_{j}$ has trivial centralizer in $\hat{G}$ and adjoint Swan conductor

$$
b\left(\varphi_{j}, \hat{\mathfrak{g}}\right)=\frac{\operatorname{dim} \hat{\mathfrak{g}}-\operatorname{dim} \hat{\mathfrak{g}}^{J}}{2(p+1)}=\frac{p-1}{2}=\ell_{\vartheta},
$$

in accordance with (7.3) and (7.4).

7.5. Examples in $G_{2}$. Let $G$ be a group over $k$ of type $G_{2}$, with simple roots $\alpha_{1}, \alpha_{2}$, where $\alpha_{2}$ is short. Let $x=\frac{1}{3} \check{\omega}_{1}$, where the $\check{\omega}_{i}$ are the fundamental coweights dual to $\alpha_{i}$. The Kac coordinates of $x$ are given in the diagram

$$
11 \Rightarrow 0 \text {, }
$$

and $\mathrm{G}_{x}=\mathrm{GL}_{2}(U)$, where $U$ is a two-dimensional vector space over $\mathfrak{F}$.

We will assume that $p \neq 3$. (The case $p=3$ is more complicated but can be handled by a modification of our present methods.) Since $p \neq 3$, the $\mathrm{G}_{x^{-}}$ representation $\check{\mathrm{V}}_{x}=\check{\mathrm{V}}_{x, 1 / 3}$ is given by

$$
\check{\mathrm{V}}_{x} \simeq\left[\operatorname{det}^{2} \otimes P_{3}(U)\right] \oplus\left[\operatorname{det}^{-1} \otimes P_{0}(U)\right],
$$

where $P_{d}(U)$ is the space of homogeneous polynomials of degree $d$ on $U$, with the natural action of $\mathrm{G}_{x}=\mathrm{GL}(U)$. Choosing coordinates, we may regard a vector in $\check{\mathrm{V}}_{x}$ as a pair $(f, z)$, where $f=f(x, y)$ is a binary cubic polynomial over $\mathfrak{F}$ and $z \in \mathfrak{F}$. Using the Hilbert-Mumford criterion [28, p.41], one checks that $(f, z)$ is stable if and only if $z \neq 0$ and $f$ has three distinct roots in the projective line.

If $p>3$, then $x$ arises from the square of a Coxeter element $c \in W\left(G_{2}\right)$ (see Section 5.1), and a Cartan subspace $\mathfrak{c}$ is a line in $\check{\mathrm{V}}_{x}$ on which the little Weyl group $W(\mathfrak{c}, \theta) \simeq\langle c\rangle \simeq \boldsymbol{\mu}_{6}$ acts as multiplication by sixth roots of unity (here $\theta$ is a lift of

\footnotetext{
${ }^{3}$ The element $\sigma_{j}$ belongs to the class in ${ }^{L} G$ of "Macdonald-Kac" elements, with Kac coordinates $2 \Rightarrow 1 \quad 1 \cdots 1 \quad 1 \Rightarrow 1$.
} 
$c^{2}$ in $\mathrm{G}=G_{2}(\mathfrak{F})$ ). The results of Vinberg-Levy theory (recalled in Section 5) and Theorem 4.1 show that each nonzero closed $\mathrm{G}_{x}$-orbit in $\check{\mathrm{V}}_{x}$ meets $\mathfrak{c}$ in exactly six points which are permuted freely and transitively by $W(\mathfrak{c}, \theta)$ and that the algebra of $\mathrm{G}_{x}$-invariant polynomials on $\check{\mathrm{V}}_{x}$ is generated by a degree-six polynomial on $\check{\mathrm{V}}_{x}$.

Indeed, the polynomial given by

$$
\Delta(f, z)=\operatorname{disc}(f) \cdot z^{2}
$$

where $\operatorname{disc}(f)$ is the discriminant of $f$, is invariant under $\mathrm{G}_{x}$ and has degree six; for if we write $f(x, y)=a x^{3}+b x^{2} y+c x y^{2}+d y^{3}$, then $\operatorname{disc}(f)$ becomes the quartic polynomial

$$
\operatorname{disc}(f)=b^{2} c^{2}+18 a b c d-4 a c^{3}-4 d b^{3}-27 a^{2} d^{2} .
$$

Since $\operatorname{disc}(f) \neq 0$ precisely when $f$ has distinct zeros in the projective line, our discussion above shows that the stable points in $\check{\mathrm{V}}_{x}$ are precisely those where $\Delta \neq 0$. Moreover, the level set $\Delta^{-1}(h)$ is a single $\mathrm{G}_{x}$-orbit, for each nonzero value $h \in \mathfrak{F}$. Indeed, if $\Delta(v)=h=\Delta\left(v^{\prime}\right)$, then $v, v^{\prime}$ are stable and hence are $\mathrm{G}_{x}$-conjugate to some points $v_{0}, v_{0}^{\prime} \in \mathfrak{c}$. Since $\operatorname{dim} \mathfrak{c}=1$, we have $v_{0}^{\prime}=t v_{0}$ for some $t \in \mathfrak{F}^{\times}$, so that $0 \neq \Delta\left(v_{0}\right)=\Delta\left(v_{0}^{\prime}\right)=t^{6} \cdot \Delta\left(v_{0}\right)$ and we have $t \in \boldsymbol{\mu}_{6}$. Thus there exists $w \in W(\mathfrak{c}, \theta)$ such that $w \cdot v_{0}^{\prime}=v_{0}$, so $v, v^{\prime}$ are $\mathrm{G}_{x}$-conjugate, as claimed. B. Gross has shown us how this classification of stable orbits can also be proved using the classical theory of binary cubic forms.

The isotropy group in $\mathrm{G}_{x}$ of a stable vector $(f, z)$ is $\operatorname{Stab}_{\mathrm{G}_{x}}(f) \cap \mathrm{SL}(U)$. Also, $\operatorname{Stab}_{G_{x}}(f)$ is the symmetric group $S_{3}$ (permuting the three roots of $f$ ), on which the determinant equals the sign character of $S_{3}$. It follows that the isotropy groups in $\mathrm{G}_{x}$ of stable points are isomorphic to $\boldsymbol{\mu}_{3}$ over $\mathfrak{F}$. Hence the isotropy group $A_{x, \lambda}$ in $\mathrm{G}_{x}(\mathfrak{f})$ of a stable rational functional $\lambda=(f, z) \in \check{\mathrm{V}}(\mathfrak{f})$ is a subgroup of $\boldsymbol{\mu}_{3}$.

The parameter $\varphi_{\lambda}$, from the construction of Section 7.3, has image in ${ }^{L} G=\hat{G}=$ $G_{2}(\mathbb{C})$ of the form

$$
D_{1} \rtimes\langle\hat{\sigma}, \hat{\tau}\rangle \subset N_{\hat{G}}(\hat{T}),
$$

with wild inertia group $D_{1} \subset \hat{T}[p]$ of order $p^{r}$ (where $r \in\{1,2\}$ is the order of $p$ in $\left.\mathbb{Z} / 3^{\times}\right)$, inertia group $D_{0}=D_{1} \rtimes\langle\hat{\sigma}\rangle$, and Frobenius image $\hat{\tau}$.

Now $\sigma$ is a Coxeter element in the Weyl group of the subgroup $\hat{H} \simeq \mathrm{SL}_{3}(\mathbb{C})$ containing $\hat{T}$ and we have

$$
\operatorname{im} \varphi_{\lambda} \subset N_{\hat{G}}(\hat{T}) \subset N_{\hat{G}}(\hat{H}),
$$

where $N_{\hat{G}}(\hat{H})=\hat{H} \cdot 2$ is the normalizer of $\hat{H}$, and

$$
A_{\varphi_{\lambda}}=\hat{Z}^{\tau}
$$

the fixed points of $\tau$ acting on the center $\hat{Z}$ of $\hat{H}$ by conjugation. One checks that

$$
\Delta(\lambda) \in \mathfrak{f}^{\times 2} \quad \Leftrightarrow \quad A_{x, \lambda}=\boldsymbol{\mu}_{3} \quad \Leftrightarrow \quad \hat{Z}^{\tau}=\hat{Z} \quad \Leftrightarrow \quad \operatorname{im} \varphi_{\lambda} \subset \hat{H} .
$$

It follows that for any character $\rho: A_{\varphi_{\lambda}} \rightarrow \mathbb{C}^{\times}$the pair $\left(\varphi_{\lambda}, \rho\right)$ may be regarded as a simple wild parameter [14, Section 6] for the group $H(k)$, where

$$
H= \begin{cases}\mathrm{PGL}_{3} & \text { if } \hat{Z}^{\tau}=\hat{Z} \text { and } \rho=\operatorname{triv} \\ \mathrm{PD}_{3} & \text { if } \hat{Z}^{\tau}=\hat{Z} \text { and } \rho \neq \operatorname{triv} \\ \mathrm{PU}_{3} & \text { if } \hat{Z}^{\tau}=1 \text { and } \rho=\operatorname{triv} \text { (necessarily) }\end{cases}
$$

Thus, $\left(\varphi_{\lambda}, \rho\right)$ also corresponds to a simple supercuspidal representation $\pi_{H}\left(\varphi_{\lambda}, \rho\right)$ of $H(k)$. 
On $G_{2}(k)$, the induced representation

$$
\pi_{x}(\lambda)=\bigoplus_{\rho \in \operatorname{Irr}\left(\hat{Z}^{\tau}\right)} \pi_{x}(\lambda, \rho)
$$

is a sum of three or one irreducible epipelagic supercuspidal representations, according to whether the equivalent conditions (7.16) hold or not. The conjectures of [15] predict that $\pi_{x}(\lambda, \rho)$ is a lift of $\pi_{H}\left(\varphi_{\lambda}, \rho\right)$ under the exceptional theta correspondence for $G_{2} \times H$ arising from the minimal representation of an unramified group of type $E_{6}\left[\frac{4}{4}\right.$

Now assume $p=2$, a torsion prime for $G_{2}$. The description (7.15) of $\check{\mathrm{V}}_{x}$ in terms of binary cubics is still valid, but now the discriminant becomes a square:

$$
\operatorname{disc}\left(a x^{3}+b x^{2} y+c x y^{2}+d y^{3}\right)=(a d+b c)^{2},
$$

so we now have a cubic polynomial invariant

$$
\delta(f, z)=(a d+b c) z,
$$

with $\delta^{2}=\Delta$. The stable points in $\check{\mathrm{V}}_{x}$ are those with nonzero value of $\delta$. But the sign character is trivial when $p=2$, so the isotropy groups of stable points are now isomorphic to $S_{3}$ over $\mathfrak{F}$. If $\lambda \in \check{\mathrm{V}}_{x}(\mathfrak{f})$ is a stable rational point, then the rational stabilizer $A_{x, \lambda}$ is a subgroup of $S_{3}$.

Consider the case $k=\mathbb{Q}_{2}$, where $\mathrm{G}_{x}(\mathfrak{f})=\mathrm{GL}_{2}\left(\mathbb{F}_{2}\right)=S_{3}$. There are six stable vectors in $\check{\mathrm{V}}_{x}\left(\mathbb{F}_{2}\right)$; they correspond to elements of $\mathrm{GL}_{2}\left(\mathbb{F}_{2}\right)$ via $a x^{3}+b x^{2} y+c x y^{2}+$ $d y^{3} \leftrightarrow\left[\begin{array}{ll}b & a \\ d & c\end{array}\right]$, under which the action of $\mathrm{GL}_{2}\left(\mathbb{F}_{2}\right)$ on the set of stable vectors corresponds to its action on itself by conjugation. Thus the $\mathrm{GL}_{2}\left(\mathbb{F}_{2}\right)$-orbits of stable vectors in $\check{\mathrm{V}}_{x}\left(\mathbb{F}_{2}\right)$ are in canonical bijection with the conjugacy classes in $S_{3}$.

Up to conjugacy, $G_{2}(\mathbb{C})$ has a unique closed subgroup isomorphic to $\mathrm{SO}_{3}(\mathbb{C}) \times$ $S_{3}$; the first factor is the image of the subregular homomorphism $\varphi^{\prime}: \mathrm{SL}_{2}(\mathbb{C}) \rightarrow$ $G_{2}(\mathbb{C})$, and the second factor $S_{3}$ is the full centralizer of the first factor. Let $\varphi: \operatorname{Gal}\left(\overline{\mathbb{Q}}_{2} / \mathbb{Q}_{2}\right) \rightarrow \mathrm{SO}_{3}(\mathbb{C})$ be the unique simple wild parameter [14, 6.3]. Then $\varphi$ has image $S_{4}$ and inertial image $A_{4}$. One can check 5 that the centralizer of this $S_{4}$ in $G_{2}(\mathbb{C})$ is just the $S_{3}$ centralizing this $\mathrm{SO}_{3}(\mathbb{C})$. For each $s \in S_{3}$ we have an unramified twist

$$
\varphi_{s}=\varphi \times \chi_{s}: \operatorname{Gal}\left(\overline{\mathbb{Q}}_{2} / \mathbb{Q}_{2}\right) \rightarrow \mathrm{SO}_{3}(\mathbb{C}) \times S_{3},
$$

where $\chi_{s}$ is the unramified character such that $\chi_{s}($ Frob $)=s$. The centralizer $A_{\varphi_{s}}=C_{\hat{G}}\left(\operatorname{im} \varphi_{s}\right)=C_{S_{3}}(s) \simeq A_{x, \lambda}$, where $\lambda \in \check{\mathrm{V}}_{x}\left(\mathbb{F}_{2}\right)$ is a stable vector whose orbit corresponds to the class of $s$, as above.

The image of wild inertia under $\varphi_{s}$ is the Klein subgroup $K_{4}<A_{4}$, whose centralizer in $\hat{G}$ is $\hat{T}$ extended by inversion. The Swan conductor does not see this extension: we still have

$$
b\left(\varphi_{s}\right)=\frac{\operatorname{dim} \hat{\mathfrak{g}}-\operatorname{dim} \hat{\mathfrak{t}}}{\left[A_{4}: K_{4}\right]}=4=\operatorname{dim} \mathrm{G}_{x} .
$$

Thus the expected equalities (7.3) and (7.4) continue to hold when $p=2$.

\footnotetext{
${ }^{4}$ See also [12], which verifies the conjectures in 15 for nonsupercuspidal representations of $H$.

${ }^{5}$ Consider the restriction of the irreducible seven-dimensional representation of $G_{2}(\mathbb{C})$ to $\mathrm{SO}_{3}(\mathbb{C})$.
} 
Denoting stable orbits in $\check{\mathrm{V}}_{x}\left(\mathbb{F}_{2}\right)$ by partitions of 3 , we have three induced representations decomposing into irreducible epipelagic supercuspidal representations of $G_{2}\left(\mathbb{Q}_{2}\right)$ as follows:

$$
\begin{aligned}
\pi_{x}(111) & =\pi_{x}(111, \text { triv }) \oplus 2 \cdot \pi_{x}(111, \text { refl }) \oplus \pi_{x}(111, \text { sgn }), \\
\pi_{x}(21) & =\pi_{x}(21, \text { triv }) \quad \oplus \pi_{x}(21, \text { sgn }), \\
\pi_{x}(3) & =\pi_{x}(3, \text { triv }) \quad \oplus \pi_{x}(3, \omega) \quad \oplus \pi_{x}\left(3, \omega^{-1}\right),
\end{aligned}
$$

where $\omega: \mu_{3} \rightarrow \mathbb{C}^{\times}$is a character of order three.

We note this is parallel to the three unipotent $L$-packets attached to the unramified discrete parameters

$$
\varphi_{s}^{\prime}=\varphi^{\prime} \times \chi_{s}: \mathrm{SL}_{2}(\mathbb{C}) \times \operatorname{Gal}\left(\overline{\mathbb{Q}}_{2} / \mathbb{Q}_{2}\right) \longrightarrow \mathrm{SO}_{3}(\mathbb{C}) \times S_{3} \subset G_{2}(\mathbb{C}),
$$

as in 25] and 29].

\section{Semistable Vectors}

An irreducible admissible representation of $G(k)$ has positive depth if it contains no nonzero vectors invariant under $G(k)_{x, 0+}$, for any $x \in \mathcal{B}(G, k)$. In [26], Moy and Prasad proved the fundamental result that for every irreducible admissible positive-depth representation $\pi$ of $G(k)$ there is a pair $(x, r) \in \mathcal{A}(S, k) \times \mathbb{R}_{>0}$ such that $\pi$ contains a character $\chi_{\lambda}$ of $G(k)_{x, r} / G(k)_{x, r+}$ such that $\lambda \in \check{\mathrm{V}}_{x, r}(\mathfrak{f})$ is semistable. (This is a reformulation of the original statement in [26]; see [11, Lemma 4.1.2].) It is known that if $\check{\mathrm{V}}_{x, r}$ has semistable vectors for the action of $\mathrm{G}_{x}$, then $r$ is a rational number [2, 3.2].

The aim of this section is to classify the pairs $(x, r)$ for which $\check{\mathrm{V}}_{x, r}(\mathfrak{f})$ contains semistable vectors under $\mathrm{G}_{x}$. We will use Theorem 4.1 and [32, Thm. 29]. The latter result is only valid for inner automorphisms of the Lie algebra $\mathfrak{L}$. This means in our present application of it that we must assume $G$ is split over $K$. As we have seen, there are also restrictions on the residual characteristic $p$ which depend on the denominator $m$ of $r$. But $m$ is not known a priori, so we must first find a universal bound on $m$ which is independent of $p$. For this issue there is no distinction between $\check{\mathrm{V}}_{x, r}$ and $\mathrm{V}_{x, r}$, so we shall consider the latter.

8.1. A necessary condition for semistability. In this section we show, without any conditions on $p$, that if $\check{\mathrm{V}}_{x, r}$ contains semistable vectors, then the denominator of $r$ is bounded by the Coxeter number $h$ of $G$.

We will assume $G$ is split over $K$ (since that will eventually be required anyway) and that $G$ is absolutely simple. In particular $T$ is now a maximal torus in $G$ and the root system $R$ is irreducible.

We have $\vartheta=1$ and we now write $\mathcal{A}:=\mathcal{A}(T, K)$. Using the basepoint $x_{0} \in \mathcal{A}$, we identify $\mathcal{A}=\mathbb{R} \otimes \check{X}$. Thus we identify each root $\alpha \in R$ with the affine function $\tilde{\alpha}$, and the system of affine $K$-roots is now written as

$$
\Psi=R+\mathbb{Z}=\{\alpha+n: \alpha \in R, n \in \mathbb{Z}\} .
$$

We can also scale points in $\mathcal{A}$ with respect to $x_{0}$. That is, for $t \in \mathbb{R}$ and $x \in \mathcal{A}$, we define $t x=t\left(x-x_{0}\right)+x_{0}$.

For each $(x, r) \in \mathcal{A} \times \mathbb{R}$, the set

$$
R_{x, r}=\{\alpha \in R: \alpha(x) \in r+\mathbb{Z}\}
$$


is the set of nonzero weights of $\mathrm{V}_{x, r}$ under the torus $\mathrm{T}:=T(K)_{0} / T(K)_{0+}$ (Section 2.3)

We now fix $(x, r) \in \mathcal{A} \times \mathbb{Q}$ and write $r=k / m$ with $\operatorname{gcd}(k, m)=1$. We will prove:

Proposition 8.1. Assume that $\mathrm{V}_{x, r}$ has semistable vectors under $\mathrm{G}_{x}$. Then $m \leq h$, the Coxeter number of $G$.

Before beginning the proof, we need a few more preparatory remarks.

First, suppose that $R_{x, r}$ is empty. In this case, since $\mathrm{V}_{x, r}$ is nonzero (by the definition of semistability), we must have $\mathrm{V}_{x, r}=T(K)_{r} / T(K)_{r+} \neq 0$, which forces $r \in \mathbb{Z}$, the valuation group of $K$. So in this case we have $m=1 \leq h$. Henceforth we assume $R_{x, r}$ is nonempty.

Next, in contrast to Proposition [3.2 the hypotheses of Proposition 8.1 impose no obvious relation between $x$ and $m$. In fact, $x$ need not even be rational with respect to $\Psi$. However, $x$ is rational with respect to a certain affine root subsystem of $\Psi$, which we shall soon describe.

For any root subsystem $R^{\prime} \subset R$, the set

$$
\Psi^{\prime}:=R^{\prime}+\mathbb{Z}=\left\{\alpha+n: \alpha \in R^{\prime}, n \in \mathbb{Z}\right\}
$$

is an affine root subsystem of $\Psi$. The collection of hyperplanes $\left\{\psi^{-1}(0): \psi \in \Psi^{\prime}\right\}$ partitions $\mathcal{A}$ into $\Psi^{\prime}$-facets [5, V.2]. Note that $\Psi^{\prime}$-facets are unbounded if $R^{\prime}$ has smaller rank than $R$.

Given $y \in \mathcal{A}$, let $\mathcal{C}^{\prime}$ be any $\Psi^{\prime}$-alcove containing $y$ in its closure, and let $\Pi^{\prime} \subset \Psi^{\prime}$ be the set of $\psi \in \Psi^{\prime}$ whose vanishing hyperplanes $\psi^{-1}(0)$ are the walls of $\mathcal{C}^{\prime}$. We say that $y$ is a $\Psi^{\prime}$-barycenter if all $\psi \in \Pi^{\prime}$ not vanishing at $y$ take the same value. From [5, p. 80] it follows that this condition is independent of the choice of $\mathcal{C}^{\prime}$ containing $y$ in its closure. The $\Psi^{\prime}$-barycenters contained in a given $\Psi^{\prime}$-facet form an affine space of dimension equal to the co-rank of $R^{\prime}$ in $R$.

We say that a point $y \in \mathcal{A}$ is $\Psi^{\prime}$-rational if $\psi(y) \in \mathbb{Q}$ for all $\psi \in \Psi^{\prime}$. When this holds, we define the $\Psi^{\prime}$-order of $y$ to be the smallest positive integer $n$ such that $\psi(y) \in \frac{1}{n} \mathbb{Z}$ for all $\psi \in \Psi^{\prime}$.

Returning now to $\mathrm{V}_{x, r}$ with $r=k / m$ in lowest terms, let $j$ be a multiplicative inverse of $k$ modulo $m$, let $y$ be the scaled point

$$
y:=j x,
$$

and consider $R_{y, 1 / m}=\left\{\alpha \in R: \alpha(y) \in \frac{1}{m}+\mathbb{Z}\right\}$. One checks that

$$
R_{x, r} \subset R_{y, 1 / m} \text {. }
$$

Since we have assumed $R_{x, r}$ is nonempty, it follows that $R_{y, 1 / m}$ is nonempty.

Now $R_{y, 1 / m}$ is contained in the root subsystem

$$
R^{\prime}:=\left\{\alpha \in R: \alpha(y) \in \frac{1}{m} \mathbb{Z}\right\} .
$$

Let $\Psi^{\prime}=R^{\prime}+\mathbb{Z}$ be the corresponding affine root subsystem, as above. By construction we have $\Psi^{\prime}(y) \subset \frac{1}{m} \mathbb{Z}$, so $y$ is $\Psi^{\prime}$-rational of order at most $m$, and since $R_{y, 1 / m} \neq \varnothing$, it follows that there exists $\psi \in \Psi^{\prime}$ such that $\psi(y)=1 / m$. Therefore, $m$ is the $\Psi^{\prime}$-order of $y$.

Decompose $R^{\prime}$ into its irreducible components $R_{i}^{\prime}$ :

$$
R^{\prime}=R_{1}^{\prime} \cup \cdots \cup R_{t}^{\prime} .
$$

This gives a corresponding decomposition

$$
\Psi^{\prime}=\Psi_{1}^{\prime} \cup \cdots \cup \Psi_{t}^{\prime},
$$


where $\Psi_{i}^{\prime}=R_{i}^{\prime}+\mathbb{Z}$. Finally, we have a decomposition

$$
\mathcal{A}=\mathcal{A}_{0} \times \mathcal{A}_{1} \times \cdots \times \mathcal{A}_{t},
$$

such that if $\psi \in \Psi_{i}^{\prime}$ for some $1 \leq i \leq t$ and if $z=\left(z_{0}, z_{1}, \ldots, z_{t}\right) \in \mathcal{A}$, then the value $\psi(z)$ depends only on the component $z_{i}$; we denote this value by $\psi(z)=\psi\left(z_{i}\right)$. Thus, $\Psi_{i}^{\prime}$ is an affine root system on $\mathcal{A}_{i}$, whose gradient root system $R_{i}^{\prime}$ is irreducible.

Let $y=\left(y_{0}, y_{1}, \ldots, y_{t}\right)$, and let $m_{i}$ be the $\Psi_{i}^{\prime}$-order of $y_{i}$, for $1 \leq i \leq t$. Since, for each $\psi \in \Psi_{i}^{\prime}$, we have $\psi\left(y_{i}\right)=\psi(y) \in \frac{1}{m} \mathbb{Z}$, it follows that

$$
m_{i} \leq m \text {. }
$$

Let $I=\left\{i: 1 \leq i \leq t, R_{i}^{\prime} \cap R_{y, 1 / m} \neq \varnothing\right\}$. Note that $I$ itself is nonempty, since $R_{y, 1 / m}$ is nonempty. Let $i \in I$ and let $\alpha \in R_{i}^{\prime} \cap R_{y, 1 / m}$. Then $\alpha(y)=1 / m-n$ for some $n \in \mathbb{Z}$, so the affine root $\psi=\alpha+n$ belongs to $\Psi_{i}^{\prime}$ and we have $\psi(y)=1 / \mathrm{m}$. Combined with (8.2), it follows that

$$
m_{i}=m, \quad \text { for any } \quad i \in I .
$$

We claim there exists $i \in I$ for which $y_{i}$ is a $\Psi_{i}^{\prime}$-barycenter. For if not, then by (8.3), we can apply Lemma 3.1 to $y_{i}, \Psi_{i}^{\prime}$ for every $i \in I$. Hence there exists $\check{\lambda}_{i}$ in the co-root lattice of $R_{i}^{\prime}$ such that $\left\langle\alpha, \check{\lambda}_{i}\right\rangle>0$ for all $\alpha \in R_{i}^{\prime} \cap R_{y, 1 / m}$. As $\left\langle\beta, \check{\lambda}_{i}\right\rangle=0$ for all $\beta \in R_{j}^{\prime}$ with $j \neq i$, it follows that the sum

$$
\check{\lambda}:=\sum_{i \in I} \check{\lambda}_{i}
$$

satisfies $\langle\alpha, \check{\lambda}\rangle>0$ for all $\alpha \in R_{y, 1 / m}$. By (8.1) this also holds for all $\alpha \in R_{x, r}$, contradicting the assumption that $\mathrm{V}_{x, r}$ has semistable vectors.

Choose $i \in I$ such that $y_{i}$ is a $\Psi_{i}^{\prime}$-barycenter. The $\Psi_{i}^{\prime}$-order $m_{i}=m$ of $y_{i}$ is at most the Coxeter number $h_{i}$ of $R_{i}^{\prime}$. From [5, V.6.2] or inspection of cases, we have $h_{i} \leq h$. Thus, $m \leq h$, and Proposition 8.1 is proved.

8.2. Existence of semistable vectors. In this section we determine the pairs $(x, r)$ such that $\check{\mathrm{V}}_{x, r}$ contains semistable vectors, under the assumption that $G$ is split over an unramified extension of $k$ and that the residual characteristic $p$ is greater than the Coxeter number $h$ of $G$.

We need one more preliminary result.

Lemma 8.2. Let $\theta$ be an inner automorphism of $\mathfrak{L}$, whose order $m$ is prime to $p$, let $\zeta$ be a root of unity of order $m$, and let $\mathfrak{c} \subset \mathfrak{L}(\theta, \zeta)$ and $\mathfrak{c}^{\prime} \subset \mathfrak{L}\left(\theta^{j}, \zeta\right)$ be Cartan subspaces, where $(j, m)=1$. Then $\operatorname{dim} \mathfrak{c}=\operatorname{dim} \mathfrak{c}^{\prime}$.

Proof. There is a Cartan subalgebra $\mathfrak{s}$ of $\mathfrak{L}$ such that $\theta(\mathfrak{s})=\mathfrak{s}$ and $\mathfrak{c}=\mathfrak{s} \cap \mathfrak{L}(\theta, \zeta)$ [32, 3.1]. The restriction $\sigma:=\left.\theta\right|_{\mathfrak{s}}$ is an element of the Weyl group $W(\mathfrak{s})$ of $\mathfrak{s}$, and $\sigma^{j}=\left.\theta^{j}\right|_{\mathfrak{s}}$. The group algebra of $W(\mathfrak{s})$ splits over $\mathbb{Q}$, so $\sigma$ and $\sigma^{j}$ are conjugate in $W(\mathfrak{s})$. Hence the $\zeta$-eigenspaces $\mathfrak{c}=\mathfrak{s}(\sigma, \zeta)$ and $\mathfrak{s}\left(\sigma^{j}, \zeta\right)$ have the same dimension. Since $\mathfrak{s}\left(\sigma^{j}, \zeta\right)$ is abelian and consists of semisimple elements in $\mathfrak{L}$, it is contained in a Cartan subspace of $\mathfrak{L}\left(\theta^{j}, \zeta\right)$, so we have $\operatorname{dim} \mathfrak{c} \leq \operatorname{dim} \mathfrak{c}^{\prime}$.

Let $k$ be a multiplicative inverse of $j$ modulo $m$. Replacing the pair $\left(\theta, \theta^{j}\right)$ by $\left(\theta^{j}, \theta^{j k}\right)=\left(\theta^{j}, \theta\right)$ in the above argument, we get $\operatorname{dim} \mathfrak{c}^{\prime} \leq \operatorname{dim} \mathfrak{c}$ and the lemma is proved.

Remark. The automorphisms $\theta$ and $\theta^{j}$ need not be conjugate in $\operatorname{Aut}(\mathfrak{L})$. 
Let $\ell$ be the absolute rank of $G$. For any proper subset $J \subsetneq[0, \ell]$ let $\Delta_{J}=$ $\left\{\alpha_{j}: j \in J\right\}$ and $\check{\Delta}_{J}=\left\{\check{\alpha}_{j}: j \in J\right\}$ be the corresponding sets of simple roots and co-roots; these generate root subsystems $R_{J} \subset R$ and $\check{R}_{J} \subset \check{R}$, respectively. Let $\Delta_{J}^{\perp} \subset \mathbb{R} \otimes \check{X}$ be the annihilator of $\Delta_{J}$ in the real vector space $\mathbb{R} \otimes \check{X}$ and let $\check{\rho}_{J} \in \frac{1}{2} \check{X}$ be one-half of the sum of the co-roots $\check{\alpha} \in \check{R}_{J}$ which are positive with respect to $\check{\Delta}_{J}$.

Let $W_{J}$ be the subgroup of $W$ generated by the reflections from $\Delta_{J}$. The ring of $W_{J}$-invariant polynomials on the reflection representation of $W_{J}$ is a polynomial ring with homogeneous generators $f_{1}, \ldots, f_{|J|}$ whose degrees are uniquely determined; these are the degrees of $W_{J}$.

Theorem 8.3. Let $r=k / m \geq 0$ be a rational number (in lowest terms) and let $x \in \mathcal{A}$. Then $\check{\mathrm{V}}_{x, r}$ has semistable vectors under $\mathrm{G}_{x}$ if and only if there exists a subset $J \subset[1, \ell]$ such that the following two conditions hold:

(1) $m$ divides a degree of $W_{J}$;

(2) $x$ is $W_{\text {aff }}(R)$-conjugate to an element of the affine subspace $x_{0}+\frac{1}{m} \check{\rho}_{J}+\Delta \frac{1}{J} \subset$ $\mathcal{A}$.

Proof. Suppose that $\check{\mathrm{V}}_{x, r}$ contains semistable vectors, with $r=k / m$ in lowest terms. As in the proof of Proposition 8.1. let $R^{\prime}=\left\{\alpha \in R: \alpha(x) \in \frac{1}{m} \mathbb{Z}\right\}$ and let $G^{\prime}$ be the subgroup of $G$ generated by $T$ and the root groups $U_{\alpha}$ with $\alpha \in R^{\prime}$. The representation pairs $\left(\mathrm{G}_{x}, \check{\mathrm{V}}_{x, r}\right)$ and $\left(\mathrm{G}_{x}^{\prime}, \check{\mathrm{V}}_{x, r}^{\prime}\right)$ are isomorphic, so that $\check{\mathrm{V}}_{x, r}^{\prime}$ has semistable vectors for $\mathrm{G}_{x}^{\prime}$. Passing to a suitable irreducible component of $R^{\prime}$, we may assume $R^{\prime}$ is irreducible and that $x$ has $\Psi^{\prime}$-order $m$, where $\Psi^{\prime}=R^{\prime}+\mathbb{Z}$.

Since $p>h$ and $h$ is at least the Coxeter number of $R^{\prime}$, Proposition 8.1 ensures that $p$ is large enough so that Theorem 4.1 is valid for $G^{\prime}$. Thus, $x$ gives an automorphism $\theta$ of the Lie algebra $\mathfrak{L}^{\prime}=\operatorname{Lie}\left(\mathrm{G}^{\prime}\right)$ as in (4.4), and we have an isomorphism of representation pairs

$$
\left(\mathrm{G}_{x}^{\prime}, \check{\mathrm{V}}_{x, r}^{\prime}\right) \simeq\left(\mathrm{H}^{\prime}, \mathfrak{L}^{\prime}\left(\theta, \zeta^{k}\right)\right)
$$

where $\mathrm{H}^{\prime}$ is the identity component of the group of fixed points of $\theta$ in $\mathrm{G}^{\prime}$, the analogue of $\mathrm{G}$ for $G^{\prime}$ (cf. (4.1)). Since $\mathfrak{L}^{\prime}\left(\theta, \zeta^{k}\right)=\mathfrak{L}^{\prime}\left(\theta^{j}, \zeta\right.$ ), Lemma 8.2 (applied to $\left.\mathfrak{L}^{\prime}\right)$ implies that the Cartan subspaces of $\left(\mathbf{H}^{\prime}, \mathfrak{L}^{\prime}\left(\theta, \zeta^{k}\right)\right)$ and $\left(\mathbf{H}^{\prime}, \mathfrak{L}^{\prime}(\theta, \zeta)\right)$ have the same dimension. Thus we may assume that $k=1$.

The roots in $R^{\prime}$ are precisely those of the centralizer in $G$ of $\theta^{m}$. It follows that replacing $x$ by a conjugate under $W_{\text {aff }}(R)$, we may assume that $R^{\prime}=R_{I}$, for some proper subset $I \subsetneq[0, \ell]$. Applying Theorem 29 of $\left[32\right.$ to $R_{I}$, there is a subset $J \subset I$ such that $m$ divides a degree of $W_{J}$ and $x$ is $W_{\text {aff }}\left(R_{I}\right)$-conjugate to $x_{0}+\frac{1}{m} \check{\rho}_{J}+v$, where $v \in \Delta_{J}^{\perp}$. Hence $m$ divides a degree of $W_{J_{1}}$, where $R_{J_{1}}$ is some irreducible component of $R_{J}$, and we may write $x=x_{0}+\frac{1}{m} \check{\rho}_{J_{1}}+v_{1}$, with $v_{1} \in \Delta_{J_{1}}^{\perp}$. Replacing $R_{J}$ by $R_{J_{1}}$, we may assume that $R_{J}$ is irreducible and that $x=x_{0}+\frac{1}{m} \check{\rho}_{J}+v$, with $v \in \Delta_{J}^{\perp}$.

It remains only to show that further conjugation will put $J \subset[1, \ell]$. In other words, we must show that $R_{J}$, which is a priori a parabolic root subsystem of $R_{I}$, is in fact a parabolic root subsystem of $R$. This can be checked case by case, as follows. Suppose there exists $(I, J)$ constructed as above such that $R_{J}$ is not a parabolic subsystem of $R$. Then $J$ must contain the indices of all special nodes of the affine Dynkin diagram for $G$. Since $R_{J}$ is irreducible, $R$ can only have one of the types $B_{n}, G_{2}, F_{4}, E_{7}, E_{8}$. If $J$ has type $A_{k}$, it cannot be contained in a subset of type $A_{k+1}$, lest it be parabolic. In the (few) remaining cases one finds a root 
$\alpha \in R-R_{I}$ for which $\alpha(x) \in \frac{1}{m} \mathbb{Z}$, contradicting the definition of $R_{I}$. Thus $R_{J}$ is indeed parabolic in $R$ and this completes the proof of the "only if" direction of Theorem 8.3 .

For the other direction, suppose $x=x_{0}+\frac{1}{m} \check{\rho}_{J}+v$ where $J \subset[1, \ell], m$ divides a degree of $W_{J}$, and $v \in \Delta_{J}^{\perp}$. Now, $m$ divides the degree of some component $J_{1}$ of $J$, and we can write $x=x_{0}+\frac{1}{m} \check{\rho}_{J_{1}}+v_{1}$, with $v_{1} \in \Delta_{J_{1}}^{\perp}$. Thus we reduce to the case that $J$ is irreducible. Projecting $x$ to $\mathcal{A} / \Delta_{J}^{\perp}$, we reduce to the case that $J=[1, \ell]$ and that $x=x_{0}+\frac{1}{m} \check{\rho}$, where $m$ divides a degree of $W$. The classification of positive rank gradings [24], 32] shows that $\mathfrak{L}(\theta, \zeta)$ has nonzero Cartan subspaces, where $\theta=\operatorname{Ad}(\check{\rho}(\zeta))$. From Lemma 8.2 again, it follows that $\mathfrak{L}\left(\theta^{j}, \zeta\right)=\mathfrak{L}\left(\theta, \zeta^{k}\right)$ has nonzero Cartan subspaces and hence has nonzero closed orbits. It then follows from Theorem 4.1 that $\check{V}_{x, k / m}$ has semistable vectors, completing the proof of Theorem 8.3 .

\section{ACKNOWLEDGMENTS}

This work began when the authors visited Harvard University in 2009/2010. The authors thank Benedict Gross for encouraging their work and for his valuable comments. The examples in Sections 7.4 and 7.5 were suggested by him. The authors also thank the referee, whose careful reading led to many improvements in the exposition.

\section{REFERENCES}

[1] Jeffrey D. Adler, Refined anisotropic K-types and supercuspidal representations, Pacific J. Math. 185 (1998), no. 1, 1-32, DOI 10.2140/pjm.1998.185.1. MR1653184 (2000f:22019)

[2] Jeffrey D. Adler and Stephen DeBacker, Some applications of Bruhat-Tits theory to harmonic analysis on the Lie algebra of a reductive p-adic group, Michigan Math. J. 50 (2002), no. 2, 263-286, DOI 10.1307/mmj/1028575734. MR1914065 (2003g:22016)

[3] Armand Borel, Sous-groupes commutatifs et torsion des groupes de Lie compacts connexes, Tôhoku Math. J. (2) 13 (1961), 216-240 (French). MR0147579 (26 \#5094)

[4] A. Borel and J.-P. Serre, Sur certains sous-groupes des groupes de Lie compacts, Comment. Math. Helv. 27 (1953), 128-139 (French). MR0054612 (14,948d)

[5] Nicolas Bourbaki, Lie groups and Lie algebras. Chapters 4-6, Elements of Mathematics (Berlin), Springer-Verlag, Berlin, 2002. Translated from the 1968 French original by Andrew Pressley. MR1890629 (2003a:17001)

[6] F. Bruhat and J. Tits, Groupes réductifs sur un corps local, Inst. Hautes Études Sci. Publ. Math. 41 (1972), 5-251 (French). MR0327923 (48 \#6265)

[7] F. Bruhat and J. Tits, Groupes réductifs sur un corps local. II. Schémas en groupes. Existence d'une donnée radicielle valuée, Inst. Hautes Études Sci. Publ. Math. 60 (1984), 197-376 (French). MR756316 (86c:20042)

[8] Colin J. Bushnell and Guy Henniart, The local Langlands conjecture for GL(2), Grundlehren der Mathematischen Wissenschaften [Fundamental Principles of Mathematical Sciences], vol. 335, Springer-Verlag, Berlin, 2006. MR2234120 (2007m:22013)

[9] Colin J. Bushnell and Guy Henniart Langlands parameters for epipelagic representations of $G L_{n}$, preprint 2013.

[10] Henri Carayol, Représentations supercuspidales de $\mathrm{GL}_{n}, \mathrm{C}$. R. Acad. Sci. Paris Sér. A-B 288 (1979), no. 1, A17-A20 (French, with English summary). MR522009 (80b:22023)

[11] Stephen DeBacker, Parametrizing nilpotent orbits via Bruhat-Tits theory, Ann. of Math. (2) 156 (2002), no. 1, 295-332, DOI 10.2307/3597191. MR1935848(2003i:20086)

[12] Wee Teck Gan and Gordan Savin, Endoscopic lifts from PGL $_{3}$ to $G_{2}$, Compos. Math. 140 (2004), no. 3, 793-808, DOI 10.1112/S0010437X03000678. MR2041781(2005i:22016) 
[13] Benedict H. Gross, Irreducible cuspidal representations with prescribed local behavior, Amer. J. Math. 133 (2011), no. 5, 1231-1258, DOI 10.1353/ajm.2011.0035. MR2843098 (2012i:22030)

[14] Benedict H. Gross and Mark Reeder, Arithmetic invariants of discrete Langlands parameters, Duke Math. J. 154 (2010), no. 3, 431-508, DOI 10.1215/00127094-2010-043. MR2730575 (2012c:11252)

[15] Benedict H. Gross and Gordan Savin, The dual pair $\mathrm{PGL}_{3} \times G_{2}$, Canad. Math. Bull. 40 (1997), no. 3, 376-384, DOI 10.4153/CMB-1997-045-0. MR.1464847 (98j:22012)

[16] Jochen Heinloth, Bao-Châu Ngô, and Zhiwei Yun, Kloosterman sheaves for reductive groups, Ann. of Math. (2) 177 (2013), no. 1, 241-310, DOI 10.4007/annals.2013.177.1.5. MR2999041

[17] Kaoru Hiraga, Atsushi Ichino, and Tamotsu Ikeda, Formal degrees and adjoint $\gamma$-factors, J. Amer. Math. Soc. 21 (2008), no. 1, 283-304, DOI 10.1090/S0894-0347-07-00567-X. MR2350057 (2010a:22023a)

[18] T. Kaletha, Epipelagic L-packets and rectifying characters, preprint 2012, arXiv:1209.1720

[19] Chandrashekhar Khare, Michael Larsen, and Gordan Savin, Functoriality and the inverse Galois problem. II. Groups of type $B_{n}$ and $G_{2}$, Ann. Fac. Sci. Toulouse Math. (6) 19 (2010), no. 1, 37-70 (English, with English and French summaries). MR2597780 (2011c:11085)

[20] Ju-Lee Kim, Supercuspidal representations: an exhaustion theorem, J. Amer. Math. Soc. 20 (2007), no. 2, 273-320 (electronic), DOI 10.1090/S0894-0347-06-00544-3. MR2276772 (2008c:22014)

[21] Andrew Knightly and Charles Li, Modular L-values of cubic level, Pacific J. Math. 260 (2012), no. 2, 527-563, DOI 10.2140/pjm.2012.260.527. MR3001804

[22] Alexei I. Kostrikin and Phạm Hũ'u Tiệp, Orthogonal decompositions and integral lattices, de Gruyter Expositions in Mathematics, vol. 15, Walter de Gruyter \& Co., Berlin, 1994. MR.1308713 (96f:17001)

[23] P. C. Kutzko, Mackey's theorem for nonunitary representations, Proc. Amer. Math. Soc. 64 (1977), no. 1, 173-175. MR.0442145 (56 \#533)

[24] Paul Levy, Vinberg's $\theta$-groups in positive characteristic and Kostant-Weierstrass slices, Transform. Groups 14 (2009), no. 2, 417-461, DOI 10.1007/s00031-009-9056-y. MR2504929 (2010g:17022)

[25] George Lusztig, Some examples of square integrable representations of semisimple p-adic groups, Trans. Amer. Math. Soc. 277 (1983), no. 2, 623-653, DOI 10.2307/1999228. MR694380 (84j:22023)

[26] Allen Moy and Gopal Prasad, Unrefined minimal K-types for p-adic groups, Invent. Math. 116 (1994), no. 1-3, 393-408, DOI 10.1007/BF01231566. MR1253198(95f:22023)

[27] Allen Moy and Gopal Prasad, Jacquet functors and unrefined minimal K-types, Comment. Math. Helv. 71 (1996), no. 1, 98-121, DOI 10.1007/BF02566411. MR1371680 (97c:22021)

[28] David Mumford, Stability of projective varieties, Enseignement Math. (2) 23 (1977), no. 1-2, 39-110. MR0450272 (56 \#8568)

[29] Mark Reeder, On the Iwahori-spherical discrete series for p-adic Chevalley groups; formal degrees and L-packets, Ann. Sci. École Norm. Sup. (4) 27 (1994), no. 4, 463-491. MR.1290396 (95g:22017)

[30] Mark Reeder, Torsion automorphisms of simple Lie algebras, Enseign. Math. (2) 56 (2010), no. 1-2, 3-47. MR2674853 (2012b:17040)

[31] Mark Reeder, Elliptic centralizers in Weyl groups and their coinvariant representations, Represent. Theory 15 (2011), 63-111, DOI 10.1090/S1088-4165-2011-00377-0. MR2765477 (2012b:20108)

[32] Mark Reeder, Paul Levy, Jiu-Kang Yu, and Benedict H. Gross, Gradings of positive rank on simple Lie algebras, Transform. Groups 17 (2012), no. 4, 1123-1190, DOI 10.1007/s00031012-9196-3. MR 3000483

[33] J.-P. Serre, Coordonnées de Kac, Oberwolfach Reports, 3 (2006), pp. 1787-1790.

[34] T. A. Springer, Regular elements of finite reflection groups, Invent. Math. 25 (1974), 159-198. MR0354894 (50 \#7371)

[35] Robert Steinberg, Torsion in reductive groups, Advances in Math. 15 (1975), 63-92. MR.0354892 (50 \#7369) 
[36] J. Tits, Reductive groups over local fields, Automorphic forms, representations and $L$ functions (Proc. Sympos. Pure Math., Oregon State Univ., Corvallis, Ore., 1977), Part 1, Proc. Sympos. Pure Math., XXXIII, Amer. Math. Soc., Providence, R.I., 1979, pp. 29-69. MR.546588 (80h:20064)

[37] È. B. Vinberg, The Weyl group of a graded Lie algebra, Izv. Akad. Nauk SSSR Ser. Mat. 40 (1976), no. 3, 488-526, 709 (Russian). MR0430168 (55 \#3175)

[38] Jiu-Kang Yu, Construction of tame supercuspidal representations, J. Amer. Math. Soc. 14 (2001), no. 3, 579-622 (electronic), DOI 10.1090/S0894-0347-01-00363-0. MR 1824988 (2002f:22033)

Department of Mathematics, Boston College, Chestnut Hill, Massachusetts 02467

E-mail address: reederma@bc.edu

The Institute of Mathematical Sciences, The Chinese University of Hong Kong, Shatin, N.T., Hong Kong

E-mail address: jkyu@ims.cuhk.edu.hk 